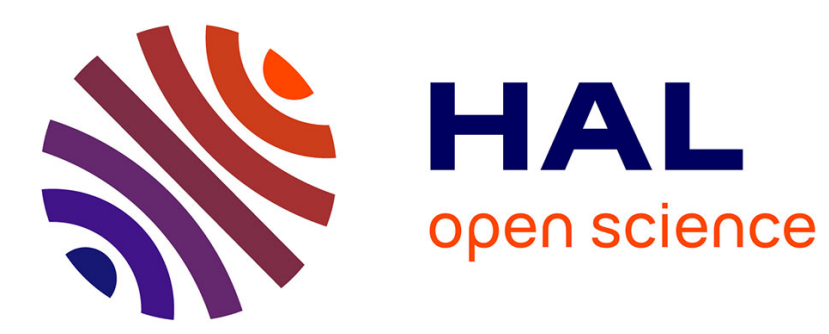

\title{
Distributed Control of Coupled Inhomogeneous Diffusion in Tokamak Plasmas
}

\author{
Bojan Mavkov, Emmanuel Witrant, Christophe Prieur
}

\section{To cite this version:}

Bojan Mavkov, Emmanuel Witrant, Christophe Prieur. Distributed Control of Coupled Inhomogeneous Diffusion in Tokamak Plasmas. IEEE Transactions on Control Systems Technology, 2019, 27 (1), pp.443-450. 10.1109/TCST.2017.2768039 . hal-01545010

\author{
HAL Id: hal-01545010 \\ https://hal.science/hal-01545010
}

Submitted on 22 Jun 2017

HAL is a multi-disciplinary open access archive for the deposit and dissemination of scientific research documents, whether they are published or not. The documents may come from teaching and research institutions in France or abroad, or from public or private research centers.
L'archive ouverte pluridisciplinaire HAL, est destinée au dépôt et à la diffusion de documents scientifiques de niveau recherche, publiés ou non, émanant des établissements d'enseignement et de recherche français ou étrangers, des laboratoires publics ou privés. 


\title{
Distributed control of coupled inhomogeneous diffusion in tokamak plasmas (full version)
}

\author{
Bojan Mavkov, Emmanuel Witrant, Christophe Prieur \\ Univ. Grenoble Alpes, CNRS, GIPSA-lab, F-38000 Grenoble, France
}

\begin{abstract}
This paper proposes novel distributed control methods for the coupled dynamics of tokamak safety factor and electron temperature. The distributed control is performed using Electron Cyclotron Resonance Heating (ECRH) actuation. The control design is based on infinite dimensional setting using Lyapunov analysis for partial differential equations (PDEs). The coupled dynamics is modeled by two 1D linearized resistive diffusion equations. We first propose a combined control of both dynamics based on a stability analysis. A composite control is then synthesized using singular perturbation theory where the fast component of the electron temperature is decoupled from the slow component induced by the magnetic field dynamics (the inverse of the safety factor being the associated controlled variable). Both control methods are evaluated using the RAPTOR simulator for tokamak devices and applied to the tokamaks TCV and ITER.
\end{abstract}

Index Terms-Distributed control methods, controlled thermonuclear fusion, Tokamak devices, partial differential equations, singular perturbation theory.

\section{INTRODUCTION}

Tokamaks are large devices using a magnetic field to confine a heat plasma in the shape of a torus. The aim of tokamak research is to build a reliable power production system using controlled thermonuclear fusion [1]. Heating of the tokamak plasma comes from the electric currents obtained from several sources. The main source of current in a tokamak is the one induced by the transformer action caused by the central ohmic coil. Other sources of current are neutral-beam injection and radio-frequency (RF) antennas. There are several plasma parameters, such as the safety factor $q$ (related to the magnetic flux) and the temperature of the electrons $T_{e}$, that are defining the plasma state. The design of a steady-state fusion reactor relies on the development of so-called advanced tokamak operation scenarios in which these plasma parameters profiles are optimized.

Simultaneous control of multiple plasma parameters profiles is a challenge, in particular because of the coupling between the magnetic flux and the pressure profiles. The safety factor is one of the key parameters to analyze the plasma magnetohydrodynamics (MHD) stability and performance. The electron temperature determines the plasma resistivity, which governs the evolution of the safety factor profile [2]. This makes these two dynamics highly coupled. In this work we focus on the simultaneous control of the poloidal magnetic flux gradient $z$ and the electron temperature $T_{e}$. These two parameters

Corresponding author: B. Mavkov (email: bojan_mavkov@yahoo.com). are modeled by nonlinear coupled resistive diffusion partial differential equations (PDEs).

The control models of the two coupled equations can be obtained by first principles or be data-driven. Data-driven models are obtained using system identification and parameter estimation techniques. Such models and simultaneous feedback control of the magnetic and kinetic plasma parameters can be found in [3], [4], [5], [6]. Control-oriented models using the first principles of physics to describe the magnetic flux profile in tokamak plasmas are proposed in [7], [8], and control-oriented models considering both the coupled poloidal flux and the electron temperature PDEs can be found in [9], [10]. Nevertheless, some of the transport coefficients are not well known and empirical models are developed for these coefficients. For example, estimation methods for heat transport coefficients are proposed in [11], [12], [13].

Numerous results were obtained on designing control algorithms for the safety factor profile using Multi Input Multi Output (MIMO) lumped models [14], [15], [16], [17], [18]. A simple control algorithm based on the singular value decomposition of the experimentally-deduced linear static response model for integrated control of $q$ and $T_{e}$ profiles is given in [19]. A control algorithm for simultaneous control of the $q$ and $T_{e}$ profiles based on a MIMO approach for finite dimensional systems based on a first principles model is presented in [10].

In this paper we focus on developing control algorithms based on infinite dimensional control theory. Several works used this method to control the safety factor profile, with different levels of simplification regarding the impact of the temperature profile (trough the plasma resistivity and the bootstrap currents). In [20], a strict Lyapunov control function for the diffusion equation of the poloidal magnetic flux gradient is computed. In this work, the resistivity coefficient is considered to be varying in space and time, and the input-tostate stability (ISS) properties of the system were examined. In [21], a Lyapunov-based control strategy using sum-ofsquares is used to maximize the bootstrap current considering a resistivity bounded between two external profiles. In [22] a proportional integral (PI) controller is developed for $\mathcal{H} \infty$ stabilization of the spatial distribution of the current profile supposing also a bounded resistivity. In this paper we consider diffusion coefficients that vary in space and time and extend the infinite dimensional Lyapunov analysis proposed by [20] to the system given by the two coupled PDEs for $z$ and $T_{e}$ profiles. We examine the stability of the nominal system and design a control strategy that improves the convergence rate to the desired equilibrium point. For this aim we proposed a 
control Lyapunov function for the coupled linearized diffusion equations with non-constant coefficients.

For the control of the coupled profiles we propose two control strategies: one that uses one control Lyapunov function for both variables and one that decouples the variables using singular perturbation theory. The first method is suitable for the small tokamaks while the second is more suitable for big scale tokamaks, for which the difference between the time scales of the two states is larger. For the second method, the model is decoupled using the theory of singularly perturbed PDE systems, in which a small parameter $\varepsilon$ is introduced: it represents the typical ratio of the thermal and resistive diffusion time scales. Singular perturbation theory is widely used in a control system theory [23], [24], [25]. Research works in boundary control of singularly perturbed partial differential equations with constant transport coefficients are introduced in [26], [27]. A composite control strategy is used, where separate controls are calculated for the slow and fast dynamics of the system. In this work, only the external heating systems are used as the constrained distributed control inputs and the induced plasma current is assumed to have given reference value.

The control methods developed in this paper are implemented on RAPTOR (RApid Plasma Transport simulatOR) [28]. RAPTOR is a control-oriented, physics-based code for simulating the 1D plasma coupled poloidal flux diffusion and the electron temperature transport. The transport equations used in this simulator are nonlinear and tuned to match the data coming from real operating tokamaks [29]. The code is used as a tool for real time control applications design, fast plasma simulation and as a real-time observer running in parallel with the plasma discharge in TCV tokamak. Our control results are evaluated on two tokamak simulations using RAPTOR: TCV and ITER. The international thermonuclear experimental reactor (ITER) is the leading research project that aims to prove the feasibility of using thermonuclear fusion for energy production. This experiment is currently under construction in Cadarache, France, and should present the transition from experimental studies of plasma physics to fusion power stations that are capable of producing sustainable energy. There exists a number of smaller currently operational tokamak devices, such as JET, DIII-D, TCV and JT-60U, that are used for experimental research. The experiments of these tokamaks have made significant progress towards realizing the goal of fusion energy.

The paper is organized as follows. In Section II the coupled PDEs for $z$ and $T_{e}$ are presented and the control problem is defined. In Section III the simplified linearized model is derived. This linearized model is used to develop the control strategies. A Lyapunov function for stability analysis is computed and used for convergence rate control in Section IV. In Section $\mathrm{V}$ the model is decoupled using singular perturbation theory and composite control is computed for the decoupled system. The control implementation is presented in Section VI. In Section VII the results from the control implemented in the nonlinear RAPTOR simulator are presented.

The main variables definitions are given in Table I.

\begin{tabular}{|c|c|c|}
\hline Variable & Symbol & Unit \\
\hline$a$ & small plasma radius & $\mathrm{m}$ \\
\hline$B_{\Psi}$ & poloidal magnetic field & $\mathrm{T}$ \\
\hline$B_{\Phi}$ & toroidal magnetic field & $\mathrm{T}$ \\
\hline$B_{0}$ & toroidal magnetic field at the center & $\mathrm{T}$ \\
\hline$I_{p}$ & total plasma current & A \\
\hline$j_{n i}$ & non inductive effective current density & $A / m^{2}$ \\
\hline$j_{b s}$ & bootstrap current density & $A / m^{2}$ \\
\hline$j_{n i}$ & auxiliary sources current density & $A / m^{2}$ \\
\hline$n_{e}$ & electron density profile & $m^{-3}$ \\
\hline$p_{e}$ & electron pressure profile & $e V m^{-3}$ \\
\hline$P_{e c}$ & electron cyclotron heating power & $W$ \\
\hline$Q_{e}$ & electron heating power density & $W / m^{3}$ \\
\hline$Q_{O H}$ & ohmic power density & $W / m^{3}$ \\
\hline$Q_{a u x}$ & auxiliary sources power density & $W / m^{3}$ \\
\hline$Q_{e i}$ & electron-ion loss power density & $W / m^{3}$ \\
\hline$Q_{\text {rad }}$ & radiation loss power density & $W / m^{3}$ \\
\hline$T_{i}$ & ions temperature profile & $e V$ \\
\hline$T_{e}$ & electrons temperature profile & $e V$ \\
\hline$V_{\text {loop }}$ & Toroidal loop voltage & V \\
\hline$x$ & normalized spatial variable & $\mathrm{m}$ \\
\hline$Z_{\text {eff }}$ & effective value of the plasma charge & \\
\hline$z$ & poloidal magnetic flux gradient & $T / m^{3}$ \\
\hline$\eta_{\| \mid}$ & plasma resistivity & $\Omega \times m$ \\
\hline$\psi$ & magnetic flux of the poloidal field & $T / m^{2}$ \\
\hline$\phi$ & toroidal magnetic flux & $T / m^{2}$ \\
\hline$q$ & safety factor & \\
\hline$\iota$ & rotational transform & \\
\hline$\chi_{e}$ & electron diffusivity & $m^{2} / s$ \\
\hline$R_{0}$ & magnetic center location & $\mathrm{m}$ \\
\hline$\rho$ & spatial variable & $\mathrm{m}$ \\
\hline$\mu_{0}$ & permeability of vacuum & $H / m$ \\
\hline
\end{tabular}

TABLE I: Most relevant physical variables and units

\section{PROBLEM DESCRIPTION}

\section{A. Magnetic flux dynamics}

For control design we are interested in the evolution of the safety factor $q$ (or its reciprocal, the rotational transform $\iota$ ), which is one of the key parameters to analyze the plasma stability and transport. The safety factor denotes the ratio of toroidal to poloidal turns for a given magnetic field surface within a tokamak. The equation that defines $q$ is:

$$
q=\frac{1}{\iota}=\frac{\partial \Phi}{\partial \Psi}=\frac{\partial \Phi / \partial x}{\partial \Psi / \partial x}=\frac{2 \pi B_{0} a^{2} x}{\partial \Psi / \partial x}
$$

where $\Psi$ is the poloidal magnetic flux, $\Phi$ is toroidal magnetic flux, $B_{0}$ the toroidal magnetic field at the center of the vacuum vessel and $x=\rho / a$ is a normalized variable of the equivalent radius of the magnetic surfaces, $\rho=\sqrt{\frac{\Phi}{\pi B_{0}}}, a$ being the small plasma radius.

We consider as controlled magnetic state the space derivative of the magnetic flux $z=\frac{\partial \Psi}{\partial x}$, since the variables of main interest ( $q, \iota$ and current profiles) depend on $z$. To design a control law with real-time capabilities, we consider the simplified one-dimensional model for $z$ (using the cylindrical approximation, which can be easily alleviated but simplifies the notations) as in [8]:

$$
\frac{\partial z}{\partial t}=\frac{\partial}{\partial x}\left(\frac{\eta_{\|}}{\mu_{0} a^{2} x} \frac{\partial}{\partial x}(x z)\right)+\frac{\partial}{\partial x}\left(\eta_{\|} R_{o} j_{n i}\right)
$$

with boundary conditions:

$$
\begin{aligned}
& z(0, t)=0, \forall t \geq 0 \\
& z(1, t)=-\frac{R_{o} \mu_{0} I_{p}(t)}{2 \pi}, \forall t \geq 0
\end{aligned}
$$


and initial condition:

$$
z(x, 0)=z_{0}(x), \forall x \in[0,1]
$$

where $R_{0}$ is the major radius of the plasma (assumed constant in time), $\mu_{0}$ is the permeability of vacuum and $\eta_{\|}(x, t)$ is the parallel electrical resistivity of the plasma. $I_{p}(t)$ is the total plasma current and $j_{n i}(x, t)$ is the non-inductive currentdensity. The non-inductive current density is obtained by combining the auxiliary sources of current density $j_{\text {aux }}(x, t)$ (current drive radio-frequency (RF) systems) and the bootstrap current density $j_{b s}(x, t)$ :

$$
j_{n i}=j_{a u x}+j_{b s}
$$

The parameters $\eta_{\|}(x, t)$ and $j_{b s}(x, t)$ are highly dependent on the dynamics of the electron temperature $T_{e}(x, t)$. The simplified models used in this work for these parameters are given in Appendix A.

The auxiliary sources are used for current drive $\left(j_{a u x}\right)$ and external heating ( $Q_{a u x}$, see next section) of the plasma. In this work they are considered as the control inputs. There are different sources that can be operated in tokamak machines, such as neutral beam injection and RF antennas operated at ion cyclotron, electron cyclotron or lower hybrid frequencies. The auxiliary current is obtained as the sum of currents produced by the RF antennas. In this work, we aim at finding the optimal profile shape of $j_{a u x}(x, t)$ that achieves the desired control action. Such shape depends on the coupling of the RF with the plasma and only a limited number of engineering parameters (such as the power, phase and position of the antennas) are available for control, thus limiting the number of degrees of freedom. We use the notation $u(t)$ to refer to all variable parameters in the auxiliary sources that can be manipulated and used as control inputs to obtain the desired $j_{\text {aux }}(x, t)$ profile. The simplified expression of $j_{\text {aux }}(x, t)$ for the configurations of the simulator used in this work is presented in Section VI.

In this paper we focus on developing a control strategy for the evolution of $z$, which can be directly related to the rotational transform $\iota(x, t)=\frac{1}{2 \pi B_{0} a^{2} x} z(x, t)$ (a parameter that is most often used as the controlled state in tokamaks).

\section{B. Electron temperature dynamics}

In tokamak plasma the transport phenomena of the electron temperature $T_{e}$ and density $n_{e}$ are coupled and modeled by a diffusion equation. This equation is obtained from simplified $1 \mathrm{D}$ energy transport and is presented as in [30], using the cylindrical approximation, as:

$$
\frac{3}{2} \frac{\partial\left(n_{e} T_{e}\right)}{\partial t}=\frac{1}{a^{2}} \frac{1}{x} \frac{\partial}{\partial x}\left(x n_{e} \chi_{e} \frac{\partial T_{e}}{\partial x}\right)+Q_{e}
$$

with boundary conditions:

$$
\begin{aligned}
\frac{\partial T_{e}}{\partial x}(0, t) & =0, \forall t \geq 0 \\
T_{e}(1, t) & =T_{e, \text { edge }}(t), \forall t \geq 0
\end{aligned}
$$

and initial condition:

$$
T_{e}(x, 0)=T_{0}(x), \forall x \in[0,1]
$$

where $\chi_{e}(x, t)$ is the electron diffusivity and $Q_{e}(x, t)$ is the total electron heating power density. Note that $\chi_{e}$ and $Q_{e}$ depend on both $z$ and $T_{e}$, rendering the system coupled and nonlinear.

The electron heating energy source (the algebraic difference between the supplied and lost energies) is calculated as a sum of several contributions:

$$
Q_{e}=Q_{O H}-Q_{e i}-Q_{r a d}+Q_{a u x}
$$

where $Q_{O H}(x, t)$ is the ohmic effect power density, $Q_{e i}(x, t)$ is the electron-ion heat exchange power density and $Q_{\text {rad }}(x, t)$ is the radiation power density. Their simplified models used in this work are given in Appendix A. The auxiliary heating power density $Q_{a u x}(x, t)$ comes from the auxiliary sources that are used as control inputs. As for $j_{a u x}(x, t)$, our feedback control approach is designed to optimize the engineering inputs $u$ to achieve the desired $Q_{a u x}(x, t)$ profile. The model used for this variable depends on the considered tokamak device and it is adapted to our test case presented in Section VI. Note that the same $u$ sets both $j_{a u x}$ and $Q_{a u x}$.

The goal of this work is to design a control strategy for the coupled dynamics of the inverse of the safety factor $\iota$ and the electron temperature $T_{e}$. Even though in the test cases considered in Section VI we are limited by using only the electron cyclotron current drive (ECCD) antennas with fixed position, the generality of the control strategy can easily be extended to include other non-inductive actuators and it does not require a fixed position and shape of the actuators as in the most of the other works presented previously.

\section{LINEARIZED COUPLED DYNAMICS}

For control purpose we linearize the model at a given equilibrium state $\left(\bar{z}, \bar{T}_{e}\right)$. This equilibrium is calculated by taking the values of the plasma parameters when the inputs $\left(\bar{u}, \bar{I}_{p}\right)$ are constant during a sufficiently long time for the system to reach a steady state. An equilibrium is defined as a stationary solution of (2) and (6) as:

$$
\left\{\begin{array}{l}
0=\frac{\partial}{\partial x}\left(\frac{\bar{\eta}_{\|}}{\mu_{0} a^{2} x} \frac{\partial}{\partial x}(x \bar{z})\right)+\frac{\partial}{\partial x}\left(\bar{\eta}_{\|} R_{o} \bar{j}_{n i}\right) \\
0=\frac{1}{a^{2}} \frac{1}{x} \frac{\partial}{\partial x}\left(x n_{e} \bar{\chi}_{e} \frac{\partial \bar{T}_{e}}{\partial x}\right)+\bar{Q}_{e}
\end{array}\right.
$$

The linearized model is derived around the steady state by substituting, in the reference model from Section II, $z=\bar{z}+\tilde{z}$, $T_{e}=\bar{T}_{e}+\tilde{T}_{e}, u=\bar{u}+\tilde{u}$ and $I_{p}=\bar{I}_{p}+\tilde{I}_{p}$ using Taylor series with first order approximation. Here $\left(\bar{z}, \bar{T}_{e}, \bar{u}, \bar{I}_{P}\right)$ denotes the equilibrium point of the system and $\left(\tilde{z}, \tilde{T}_{e}, \tilde{u}, \tilde{I}_{p}\right)$ denotes the variations around this point. The model is simplified additionally by considering the following assumptions:

- the electron density profile is constant during the heat process, $n_{e}=n_{e}(x)$;

- the space variations of the electron density are neglected with respect to those of the temperature: $\partial n_{e} / \partial x \ll$ $\partial T_{e} / \partial x$

- only the auxiliary heating/current drive systems are considered as controlled inputs, while $I_{p}$ is assigned with 
a reference value, $I_{p}(t)=\bar{I}_{p}$. Note that $I_{p}(t)$ could be used as a controlled input in our framework using the methods described in [31].

Under these assumptions, the simplified linearized coupled model coming from (2) and (6) is derived as:

$$
\left\{\begin{aligned}
\frac{\partial \tilde{z}}{\partial t}= & \frac{\partial}{\partial x}\left(\frac{a_{1}(x)}{x} \frac{\partial}{\partial x}(x \tilde{z})\right)+\frac{\partial}{\partial x}\left(a_{2}(x) \tilde{T}_{e}\right) \\
& +\frac{\partial}{\partial x}\left(a_{3}(x) \frac{\partial \tilde{T}_{e}}{\partial x}\right)+\frac{\partial}{\partial x}\left(a_{4}(x) \tilde{z}\right) \\
& +\frac{\partial}{\partial x}\left(a_{5}(x) \tilde{j}_{a u x}(u, x, t)\right) \\
\varepsilon \frac{\partial \tilde{T}_{e}}{\partial t}= & \frac{1}{x} \frac{\partial}{\partial x}\left(x b_{1}(x) \frac{\partial \tilde{T}_{e}}{\partial x}\right)-b_{2}(x) \tilde{T}_{e} \\
& +\frac{1}{x} \frac{\partial}{\partial x}\left(x b_{3}(x) \tilde{z}\right)+\frac{b_{4}(x)}{x}\left(\frac{\partial}{\partial x}(x \tilde{z})\right) \\
& +b_{5}(x) \tilde{Q}_{a u x}(u, x, t)
\end{aligned}\right.
$$

with boundary conditions:

$$
\begin{aligned}
\tilde{z}(0, t) & =0, \forall t \geq 0 \\
\tilde{z}(1, t) & =0 \forall t \geq 0 \\
\frac{\partial \tilde{T}_{e}}{\partial x}(0, t) & =0, \forall t \geq 0 \\
\tilde{T}_{e}(1, t) & =\tilde{T}_{e, \text { edge }}(t), \forall t \geq 0
\end{aligned}
$$

and initial condition:

$$
\tilde{z}\left(x, t_{0}\right)=\tilde{z}_{0} ; \tilde{T}_{e}\left(x, t_{0}\right)=\tilde{T}_{e, 0}, \forall x \in[0,1]
$$

The coefficients of these PDEs are given in (50).

In this model $\varepsilon$ stands for the typical ratio between the energy confinement time and the characteristic resistive diffusion time. This parameter varies with the size of the different tokamak devices. In a small tokamak such as TCV this parameter is $\varepsilon \approx 0.07$, and in a large tokamak such as ITER this parameter is $\varepsilon \approx 0.01$, which makes the relative time constants of the dynamics and the impact of the couplings between the states to be very diverse.

\section{STABILITY ANALYSIS AND CONTROL OF THE COUPLED SYSTEM}

\section{A. Stability analysis}

The stability of the coupled dynamics is analyzed with the following Lyapunov function candidate:

$$
V\left(\tilde{z}, \tilde{T}_{e}\right)=\frac{1}{2} \int_{0}^{1}\left[\begin{array}{ll}
\tilde{z} & \tilde{T}_{e}
\end{array}\right]\left[\begin{array}{cc}
x^{2} p_{1}(x) & 0 \\
0 & \gamma x^{2} p_{2}(x)
\end{array}\right]\left[\begin{array}{c}
\tilde{z} \\
\tilde{T}_{e}
\end{array}\right] d x
$$

where $p_{1}(x)>0$ and $p_{2}(x)>0$ for $x \in[0,1]$ are polynomial functions. The candidate Lyapunov function is chosen as a weighted $L^{2}([0,1])$ norm and it is multiplied by the term $x$ to handle the singularity at $x=0$, which comes from the cylindrical representation of the system. The scaling parameter $\gamma$ is added to balance the differences of amplitude between $\tilde{z}$ and $\tilde{T}_{e}$. The stability of our coupled system of linearized equations is established with the following theorem.
Theorem 1. Suppose that for a given positive number $\alpha_{1}$, there exist polynomials $p_{1}$ and $p_{2}$ such that $p_{1}(x)>0$ and $p_{2}(x)>0$ for all $x \in[0,1]$, and

$$
A_{1}(x)+\alpha_{1} A_{2}(x) \leq 0
$$

for all $x \in[0,1]$, where:

$$
\begin{gathered}
A_{1}(x)=\left[\begin{array}{cccc}
A_{1,1}(x) & A_{1,2}(x) & A_{1,3}(x) & A_{1,4}(x) \\
A_{1,2}(x) & A_{2,2}(x) & A_{2,3}(x) & 0 \\
A_{1,3}(x) & A_{2,3}(x) & A_{3,3}(x) & A_{3,4}(x) \\
A_{1,4}(x) & 0 & A_{3,4}(x) & A_{4,4}(x)
\end{array}\right] \\
A_{2}(x)=\frac{1}{2}\left[\begin{array}{cccc}
x^{2} & 0 & 0 & 0 \\
0 & \gamma x^{2} & 0 & 0 \\
0 & 0 & 0 & 0 \\
0 & 0 & 0 & 0
\end{array}\right]
\end{gathered}
$$

and the elements in the $A_{1}(x)$ matrix are given in (18).

Then the time derivative $\dot{V}$ of $V$ defined in (14) along the solutions of (11) and (12) verifies:

$$
\begin{aligned}
\dot{V} \leq & \frac{-\alpha_{1}}{\max _{x \in[0,1]}\left(p_{1}(x), p_{2}(x)\right)} V \\
& +\int_{0}^{1} x^{2} p_{1}(x) \frac{\partial}{\partial x}\left(a_{4}(x) \tilde{j}_{a u x}(\tilde{u}, x, t)\right) \tilde{z} d x \\
& +\gamma \int_{0}^{1} \frac{x^{2} p_{2}(x)}{\varepsilon} b_{5}(x) \tilde{Q}_{a u x}(\tilde{u}, x, t) \tilde{T}_{e} d x, \forall t \geq 0
\end{aligned}
$$

Proof. See Appendix B.

The nominal stability $(\tilde{u}=0)$ is then directly obtained with the following corollary.

Corollary 1. If the conditions of Theorem 1 are satisfied, the system (11) with $\tilde{u}=0$, boundary conditions (12) and initial condition (13) is globally exponentially stable. The convergence rate of the system satisfies $V(t) \leq e^{-\beta_{1} t} V\left(\tilde{z}_{0}, \tilde{T}_{e, 0}\right)$, where $\beta_{1}=-\alpha_{1} /\left[\max _{x \in[0,1]}\left(p_{1}(x), p_{2}(x)\right)\right]$.

Proof. This result is directly obtained by setting $\tilde{u}=0$ : the variations of the auxiliary current and power density are zero $\left(\tilde{j}_{a u x}=\tilde{Q}_{a u x}=0\right)$ and from Theorem 1, the following inequality is obtained:

$$
\dot{V} \leq-\beta_{1} V, \quad \forall t \geq 0
$$

Integrating this inequality over time implies the desired inequality on $V(t)$.

\section{B. Calculation of the Lyapunov Function}

To compute the polynomial functions $p_{1}$ and $p_{2}$ involved in Theorem 1, let us consider them as Legendre polynomials. Using Legendre polynomials, the inequality (15) is formulated and solved as an LMI problem that is defined with $x$ in the range $[0,1]$. Legendre polynomials are orthogonal in the range $[-1,1]$, and the polynomials $p_{1}$ and $p_{2}$ can be expanded in this interval in terms of them as [32]:

$$
p_{1}(x)=\sum_{i=0}^{N_{1}} c_{1, i} P_{i}(x), \quad p_{2}(x)=\sum_{i=0}^{N_{2}} c_{2, i} P_{i}(x)
$$




$$
\begin{aligned}
& A_{1,1}=\frac{1}{2}\left(3 x p_{1}^{\prime}(x)+x^{2} p_{1}^{\prime \prime}(x)-p_{1}(x)\right) a_{1}(x)+\frac{1}{2} a_{1}^{\prime}(x)\left(x^{2} p_{1}^{\prime}(x)+3 x p_{1}(x)\right)-2 x p_{1}(x) a_{4}(x)-x^{2} p_{1}^{\prime}(x) a_{4}(x) \\
& A_{1,2}=-x p_{1}(x) a_{2}(x)-\frac{1}{2} x^{2} p_{1}^{\prime}(x) a_{2}(x)+\frac{\gamma}{2 \varepsilon} x b_{3}(x)\left(x p_{2}^{\prime}(x)+p_{2}(x)\right)+\frac{\gamma}{2 \varepsilon} x p_{2}(x) b_{4}(x) \\
& A_{1,3}=-\frac{1}{2} x^{2} p_{1}(x) a_{4}(x), A_{1,4}=-x p_{1}(x) a_{3}(x)-\frac{1}{2} x^{2} p_{1}^{\prime}(x) a_{3}(x) \\
& A_{2,2}=-\frac{\gamma}{\varepsilon} x^{2} p_{2}(x) b_{2}(x)+\frac{\gamma}{2 \varepsilon} b_{1}(x)\left(\left(x^{2} p_{2}^{\prime \prime}(x)+2 x p_{2}^{\prime}(x)+p_{2}(x)\right)+\frac{\gamma}{2 \varepsilon} b_{1}^{\prime}(x)\left(x^{2} p_{2}^{\prime}(x)+x p_{2}(x)\right)\right. \\
& A_{2,3}=-\frac{1}{2} x^{2} p_{1}(x) a_{2}(x)-\frac{\gamma}{2 \varepsilon} x^{2} b_{3}(x) p_{2}(x)+\frac{\gamma}{2 \varepsilon} x^{2} p_{2}(x) b_{4}(x) \\
& A_{3,3}=-x^{2} p_{1}(x) a_{1}(x), A_{3,4}=-\frac{1}{2} x^{2} p_{1}(x) a_{4}(x), A_{4,4}=-\frac{\gamma}{\varepsilon} x^{2} b_{1}(x) p_{2}(x)
\end{aligned}
$$

where $c_{1,1}, \ldots, c_{1, N_{1}}$ and $c_{2,1}, \ldots, c_{2, N_{2}}$ are constant, $P_{i}(x)$ is the $i-t h$ order Legendre polynomial, and $N_{1}$ and $N_{2}$ are the order of the Legendre polynomials for $p_{1}$ and $p_{2}$, respectively.

Sampling the interval $[0,1]$ and representing $p_{1}$ and $p_{2}$ as a sum of Legendre polynomials permit us to formulate the following LMI problem:

Maximize $\alpha_{1} \geq 0$

such that the polynomials $p_{1}(x)$ and $p_{2}(x)$ satisfy:

1) $0<p_{1}(x) \leq p_{1, \max }$ and $0<p_{2}(x) \leq p_{2, \max }, \forall x \in$ $[0,1]$

2) $A_{1}(x)+\alpha_{1} A_{2}(x) \leq 0, \forall x \in[0,1]$

This LMI problem for finding the unknown constant parameters $c_{1,1}, \ldots, c_{1, N_{1}}$ and $c_{2,1}, \ldots, c_{2, N_{2}}$ is solved using YALMIP

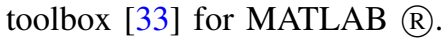

\section{Convergence rate control}

Considering the results of Theorem 1, a control strategy can be defined to accelerate the convergence rate of the system. This is done with the following corollary.

Corollary 2. If the conditions of Theorem 1 are verified, the feedback control parameters $\tilde{u}$ can be calculated to obtain the following equality:

$$
\begin{aligned}
& \int_{0}^{1} x^{2} p_{1}(x) \frac{\partial}{\partial x}\left(a_{4}(x) \tilde{j}_{a u x}(\tilde{u}, x, t)\right) \tilde{z} d x \\
& +\gamma \int_{0}^{1} \frac{x^{2} p_{2}(x)}{\varepsilon} b_{5}(x) \tilde{Q}_{a u x}(\tilde{u}, x, t) \tilde{T}_{e} d x=-\alpha_{2} V
\end{aligned}
$$

where $\alpha_{2}>0$ is a tuning parameter. Using this feedback control, the system (11) with boundary conditions (12) is globally exponentially stable and the convergence rate of the Lyapunov function satisfies $\dot{V} \leq-\beta_{2} V$, where $\beta_{2}=$ $\left(\alpha_{1}+\alpha_{2}\right) /\left[\max _{x \in[0,1]}\left(p_{1}(x), p_{2}(x)\right)\right]$.

The convergence rate is thus increased by a factor $\alpha_{2} /\left[\max _{x \in[0,1]}\left(p_{1}(x), p_{2}(x)\right)\right]$ by designing a control law that solves (implicitly) equation (21) for $\tilde{u}$.

Note that this controller is suitable for systems with commensurate time scales, such as small scale tokamaks in which there is no significant time scales difference between the fast and the slow components. This control strategy is less appropriate for large scale tokamaks, where $\epsilon$ is very small.
Indeed, using only one candidate Lyapunov function is not effective enough to control the convergence rate of a system that consists of several components with different time scales [23]. The control strategy for large tokamaks is discussed in the next section.

\section{SYSTEM DECOUPLING USING SINGULAR PERTURBATION THEORY}

In large tokamak machines, the dynamics of the evolution of $z$ is much slower than the dynamics of $T_{e}$ [3]. To deal with the two time scales we introduce the small (constant) parameter, $\varepsilon$ that represents the typical ratio between the energy confinement time and the characteristic resistive diffusion time. To apply the singular perturbation theory we divide the system into two different time scales by introducing the fast time scale $\tau=\varepsilon t$. Using this time scale we can isolate the slow variables, which are considered as fixed in the fast time scale and using static equations they are separated from the fast component.

Considering that $\varepsilon \ll 1$, the electrons temperature dynamics can be decomposed into two components: the slow component $\tilde{T}_{s}$ that evolves with the (slow) variations of the magnetic flux and the fast component $\tilde{T}_{f}$ that reacts more rapidly to inputs variation. We thus have that $\tilde{T}_{e}=\tilde{T}_{s}+\tilde{T}_{f}$. At the slow time scale, the static equation for the electron temperature is computed using the assumption that $\partial \tilde{T}_{s} / \partial \tau<<\partial \tilde{T}_{f} / \partial \tau$ in the heat equation. In this equation $\tilde{T}_{e}$ is replaced by $\tilde{T}_{s}$, which denotes the slow variation of the temperature. $\tilde{T}_{s}$ is called the quasi-steady state (QSS) and is determined by:

$$
\begin{aligned}
0= & \frac{1}{x} \frac{\partial}{\partial x}\left(x b_{1}(x) \frac{\partial \tilde{T}_{s}}{\partial x}\right)-b_{2}(x) \tilde{T}_{s}+\frac{1}{x} \frac{\partial}{\partial x}\left(x b_{3}(x) \tilde{z}\right) \\
& +\frac{b_{4}(x)}{x}\left(\frac{\partial}{\partial x}(x \tilde{z})\right)+b_{5}(x) \tilde{Q}_{a u x, s}\left(\tilde{u}_{s}, x, t\right)
\end{aligned}
$$

where $\tilde{u}_{s}$ is the slow component of the input and with boundary conditions:

$$
\frac{\partial \tilde{T}_{s}}{\partial x}(0, t)=0 ; \tilde{T}_{s}(1, t)=\tilde{T}_{e, e d g e}(t)
$$

The solution of $\tilde{T}_{s}(x, t)$ is calculated at each time instant from $\tilde{z}$ and $\tilde{u}_{s}$ in (22) using numerical methods. The evolution of 
the fast dynamics is included with a boundary layer model, obtained in the fast time scale as:

$$
\begin{aligned}
\frac{\partial \tilde{T}_{f}}{\partial \tau}= & \frac{1}{x} \frac{\partial}{\partial x}\left(x b_{1}(x) \frac{\partial \tilde{T}_{f}}{\partial x}\right)-b_{2}(x) \tilde{T}_{f} \\
& +b_{5}(x) \tilde{Q}_{a u x, f}\left(\tilde{u}_{f}, x, \tau\right)
\end{aligned}
$$

with boundary conditions:

$$
\frac{\partial \tilde{T}_{f}}{\partial x}(0, \tau)=0 ; \tilde{T}_{f}(1, \tau)=0
$$

where $\tilde{u}_{f}$ stands for the fast component of the input.

The magnetic field component $\tilde{z}$ writes in terms of $\tilde{T}_{s}$ as:

$$
\begin{aligned}
\frac{\partial \tilde{z}}{\partial t}= & \frac{\partial}{\partial x}\left(\frac{a_{1}(x)}{x} \frac{\partial}{\partial x}(x \tilde{z})\right)+\frac{\partial}{\partial x}\left(a_{2}(x) \tilde{T}_{s}\right) \\
& +\frac{\partial}{\partial x}\left(a_{3}(x) \frac{\partial \tilde{T}_{s}}{\partial x}\right)+\frac{\partial}{\partial x}\left(a_{4}(x) \tilde{z}\right) \\
& +\frac{\partial}{\partial x}\left(a_{5}(x) \tilde{j}_{a u x}\left(u_{s}, x, t\right)\right)
\end{aligned}
$$

with boundary conditions:

$$
\tilde{z}(0, t)=0 ; \tilde{z}(1, t)=0
$$

Our dynamics is thus composed of two PDEs at evolving at different time scales, (24) and (26) with their boundary and initial conditions, and one PDE that acts as an algebraic constraint, (22) with its boundary conditions. The composite control is obtained by separately calculating and combining the slow and the fast components as $\tilde{u}=\tilde{u}_{s}+\tilde{u}_{f}$.

\section{A. Slow component stability and control}

The slow component of the control is calculated by considering only the magnetic flux as setting the slow dynamics of the system (26)-(27). To compute the stability of this dynamics, the following Lyapunov function candidate is chosen:

$$
V_{s}(\tilde{z})=\frac{1}{2} \int_{0}^{1} x^{2} p_{s}(x) \tilde{z}^{2} d x
$$

The evolution of this Lyapunov function is inferred from the following theorem.

Theorem 2. Suppose that for a given positive number $\alpha_{3}$ there exists a polynomial $p_{s}$ such that $p_{s}(x)>0$ for all $x \in[0,1]$ and satisfying, for all $x \in[0,1]$,

$$
\begin{aligned}
& \frac{a_{1}(x)}{2}\left(3 x p_{s}^{\prime}(x)+x^{2} p_{s}^{\prime \prime}(x)-p_{s}(x)\right) \\
& +\frac{a_{1}^{\prime}(x)}{2}\left(x^{2} p_{s}^{\prime}(x)+3 x p_{s}(x)\right) \\
& +a_{4}(x)\left(x p_{s}(x)+\frac{x^{2}}{2} p_{1}^{\prime}(x)\right)+\frac{x^{2}}{2} a_{4}^{\prime}(x) p_{1}(x) \leq-\frac{\alpha_{3}}{2} x^{2}
\end{aligned}
$$

Then the time derivative $\dot{V}_{s}$ of the function $V_{s}$ defined by (28) verifies:

$$
\begin{aligned}
\dot{V}_{s} \leq & -\beta_{3} V_{s}+\int_{0}^{1} x^{2} p_{s}(x) \frac{\partial}{\partial x}\left(a_{2}(x) \tilde{T}_{s}+a_{3}(x) \frac{\partial \tilde{T}_{s}}{\partial x}\right) \tilde{z} d x \\
& +\int_{0}^{1} x^{2} p_{s}(x) \frac{\partial}{\partial x}\left(a_{5}(x) \tilde{j}_{a u x}\left(\tilde{u}_{s}, x, t\right)\right) \tilde{z} d x
\end{aligned}
$$

where $\beta_{3}=\frac{\alpha_{3}}{\max _{x \in[0,1]} p_{s}(x)}$

Proof. See Appendix C.

We use the dynamics (30) to design a convergence rate controller, as described in the following corollary.

Corollary 3. If the conditions of Theorem 2 are verified, the feeedback control parameters of the slow component, $\tilde{u}_{s}$ can be calculated to obtain the following relation:

$$
\begin{aligned}
& \int_{0}^{1} x^{2} p_{s}(x) \frac{\partial}{\partial x}\left(a_{2}(x) \tilde{T}_{s}+a_{3}(x) \frac{\partial \tilde{T}_{s}}{\partial x}\right) \tilde{z} d x \\
& +\int_{0}^{1} x^{2} p_{s}(x) \frac{\partial}{\partial x}\left(a_{5}(x) \tilde{j}_{a u x}\left(\tilde{u}_{s}, x, t\right)\right) \tilde{z} d x=-\alpha_{4} V_{s}
\end{aligned}
$$

where $\alpha_{4}>0$ is a tuning parameter. Using this feedback control, the system (26) with boundary conditions (27) is globally exponentially stable with a convergence rate that satisfies $\dot{V}_{s} \leq-\beta_{4} V_{s}$, where $\beta_{4}=\frac{\alpha_{3}+\alpha_{4}}{\max _{x \in[0,1]} p_{s}(x)}$.

\section{B. Fast component stability and control}

The fast component of the system has dynamics governed by (24)-(25). To compute the stability of this system (boundary layer system), the following candidate Lyapunov function is selected:

$$
V_{f}\left(\tilde{T}_{f}\right)=\frac{1}{2} \int_{0}^{1} p_{f}(x) \tilde{T}_{f}^{2} d x
$$

and its dynamics is studied in the following theorem.

Theorem 3. Suppose that for a given positive number $\alpha_{5}$ there exists a polynomial $p_{f}$ such that $p_{f}(x)>0$ for all $x \in[0,1]$ and satisfying, for all $x \in[0,1]$,

$$
\begin{aligned}
& \frac{b_{1}(x)}{2}\left(x^{2} p_{f}^{\prime \prime}(x)+2 x p_{f}^{\prime}(x)+p_{f}(x)\right) \\
& +\frac{b_{1}^{\prime}(x)}{2}\left(x^{2} p_{f}^{\prime}(x)+x p_{f}(x)\right)-x^{2} b_{2}(x) p_{f}(x) \leq-\frac{\alpha_{5}}{2} x^{2}
\end{aligned}
$$

Then the time derivative $\dot{V}_{f}$ of the function $V_{f}$ defined by (32) verifies:

$$
\dot{V}_{f} \leq-\beta_{5} V_{f}+\int_{0}^{1} x^{2} p_{f}(x) b_{5}(x) \tilde{Q}_{a u x, f}\left(\tilde{u}_{f}, x, \tau\right) \tilde{T}_{f} d x
$$

where $\beta_{5}=\frac{\alpha_{5}}{\max _{x \in[0,1]} p_{f}(x)}$.

Proof. See Appendix D.

The convergence rate of the boundary layer system is controlled using the following corollary.

Corollary 4. If the conditions of Theorem 3 are verified, the feedback control parameters of the boundary layer system $\tilde{u}_{f}$ can be calculated to obtain the following relation:

$$
\int_{0}^{1} x^{2} p_{f}(x) b_{5}(x) \tilde{Q}_{a u x, f}\left(\tilde{u}_{f}, x, \tau\right) \tilde{T}_{f} d x=-\alpha_{6} V_{f}
$$


where $\alpha_{6}>0$ is a tuning parameter. Using this feedback control, the system (24) with boundary conditions (25) is globally exponentially stable with a convergence rate that satisfies $\dot{V} \leq-\beta_{6} V$, where $\beta_{6}=\frac{\alpha_{5}+\alpha_{6}}{\max _{x \in[0,1]} p_{f}(x)}$.

Numerical algorithms to solve the functional equation (29) to find $p_{s}$ from Theorem 2 and the functional equation (33) for $p_{f}$ from Theorem 3, can be found in [20], [34]. In this work, these inequalities are scalar and they are found using Legendre polynomials and the algorithm solving the LMI problem, as the one defined in Section IV-B.

\section{Control implementation}

\section{A. Auxiliary current and power density models}

The auxiliary sources are modeled as simple weighted Gaussian distributions as proposed in [8], with the analytical parametrization defined for RAPTOR in [9]. These distributions approximate both the power and current densities. The total auxiliary current density is computed as the sum of the current densities induced by each ECCD antenna:

$$
j_{\text {aux }}=\sum_{i=1}^{n_{\text {aux }}} j_{\text {aux }, i}
$$

where $n_{\text {aux }}$ denotes the number of the auxiliary sources. Each individual auxiliary current drive is modeled as:

$$
j_{a u x, i}=\frac{\bar{T}_{e}}{n_{e}} j_{d i s, i}(x) P_{a u x, i}(t)
$$

which represents the product of the weighted Gaussian distributions $j_{d i s, i}(x)$ (given in [9]), representing the normalized reference current density deposition profiles by the input powers $P_{a u x, i}(t)$ and the current-drive efficiency $\left(\frac{\bar{T}_{e}}{n_{e}}\right)$.

Similarly, the total auxiliary power density is composed by the sum of the individuals induced by each ECCD antenna:

$$
Q_{a u x}=\sum_{i=1}^{n_{a u x}} Q_{a u x, i}
$$

The individual auxiliary power densities are modeled as:

$$
Q_{a u x, i}=Q_{d i s, i}(x) P_{a u x, i}(t)
$$

where $Q_{d i s, i}(x)$ (given in [9]) is a normalized reference power density deposition profile for the i-th auxiliary source.

In our control application, the weighted Gaussian distributions of $Q_{d i s, i}(x)$ and $j_{d i s, i}(x)$ are fixed by the choice of the position and distribution of the electron cyclotron antennas. This means that only the input powers $P_{a u x}(t)$ are available to achieve the desired control signal. Thus, the control inputs are presented as a combination of the powers of the auxiliary sources $u(t)=\left[P_{a u x, 1}(t), \ldots, P_{\text {aux }, n_{\text {aux }}}(t)\right]$.

\section{B. Calculation of the control inputs}

The control is implemented using only a limited number of actuators (the powers of the ECCD clusters $P_{a u x, i}(t)$ ) while our convergence rate controllers imply the evolution of full spatial distributions. An extra step is thus needed to optimize the engineering parameters according to the control objective.
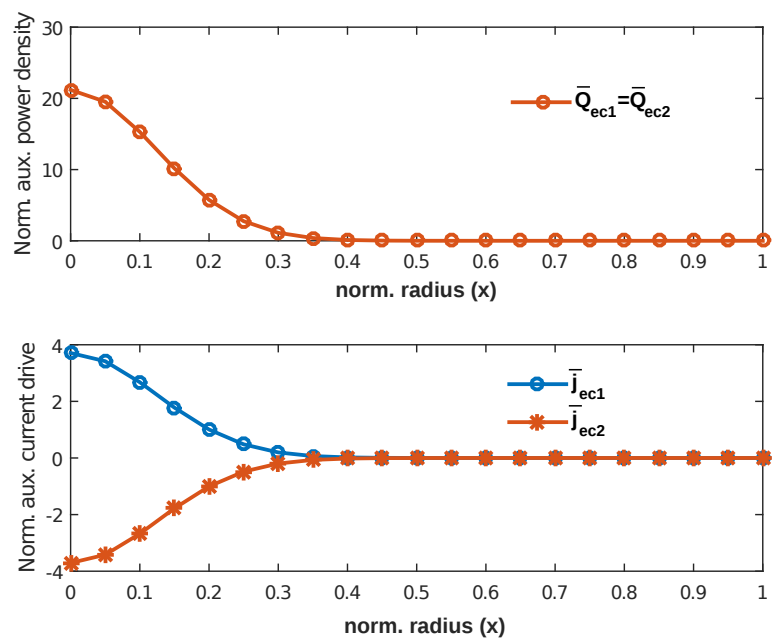

Fig. 1: Normalized auxiliary electron cyclotron deposition profiles for the TCV configuration: current-drive $\bar{j}_{\text {aux }}$ (bottom, $\times 10^{20} \frac{m^{-5} \mathrm{~A}}{\mathrm{keVW}}$ ) and power density $\bar{Q}_{a u x}$ (top, $\mathrm{m}^{-3}$ ).
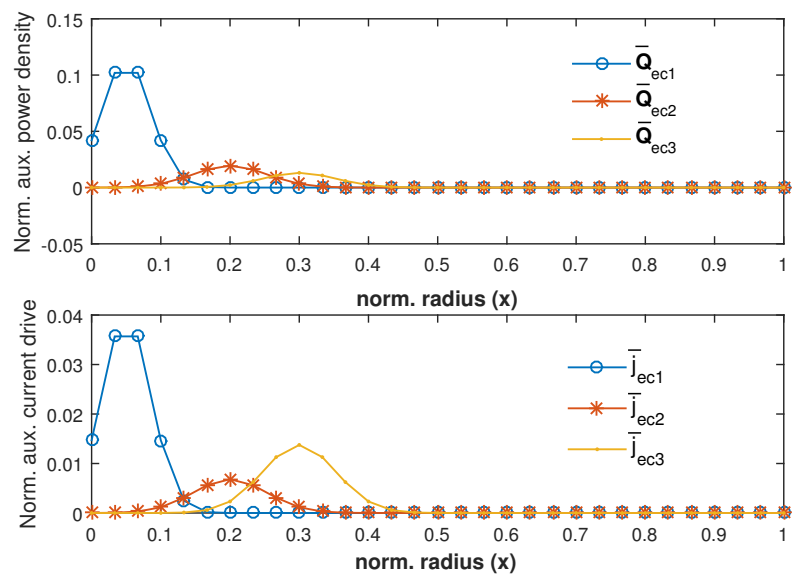

Fig. 2: Normalized auxiliary electron cyclotron deposition profiles for ITER simulation: current-drive $\bar{j}_{\text {aux }}\left(10^{20 \frac{m^{-5} \mathrm{~A}}{\mathrm{keVW}}}\right)$ and normalized auxiliary electron cyclotron power density $\bar{Q}_{\text {aux }}\left(m^{-3}\right)$.

For the practical implementation of the control strategy proposed in Corollary 2, the following optimization problem is formulated to find the engineering parameters at each time instant:

$$
\begin{aligned}
& \min _{\tilde{u}} J(\tilde{u}) \\
& \text { subject to }:- \alpha_{2} V \leq J(\tilde{u}) \leq 0 \\
& \tilde{u}_{\min } \leq \tilde{u} \leq \tilde{u}_{\max }
\end{aligned}
$$

with

$$
\begin{aligned}
J(\tilde{u})= & \int_{0}^{1} x^{2} p_{1}(x) \frac{\partial}{\partial x}\left(a_{4}(x) \tilde{j}_{a u x}(\tilde{u}, x)\right) \tilde{z} d x \\
& +\int_{0}^{1} x^{2} \frac{\gamma p_{2}(x)}{\varepsilon} b_{5}(x) \tilde{Q}_{a u x}(\tilde{u}, x) \tilde{T}_{e} d x
\end{aligned}
$$


where $\tilde{u}_{\min }$ and $\tilde{u}_{\max }$ stand for the minimum and maximum values of the allowed power for each antenna.

Remark. The convergence rate of the system in this section is calculated from a practical implementation standpoint. It takes into account the limitations of the current and of the power distribution profiles, imposed by the limitations of the auxiliary heating sources. These limitations make the equality (21) to be very restrictive and difficult to achieve. The optimization (40) solves the optimal engineering parameters to reach the desired convergence rate $\alpha_{2}$ in a less strict way. Therefore, the closed-loop system is stable and the convergence rate varies in the range: $-\beta_{1} V \leq \dot{V} \leq-\beta_{2} V$ (between the open-loop and unconstrained control values), depending on the actuators limitations.

When the system is divided into two components using singular perturbation theory (decoupled control), there are two objective functions that should be solved: one for the slow component (31) and one for the fast component (35). Combining these two functions for feedback control design, the following multi-objective optimization problem is formulated:

$$
\begin{array}{cl}
\min _{\tilde{u}=\tilde{u}_{s}+\tilde{u}_{f}} & \omega_{1} J_{s}\left(\tilde{u}_{s}\right)+\omega_{2} J_{f}\left(\tilde{u}_{f}\right) \\
\text { subject to }: & -\alpha_{4} \mathcal{V}_{s} \leq J_{s}\left(\tilde{u}_{s}\right) \leq 0 \\
& -\alpha_{6} \mathcal{V}_{f} \leq J_{f}\left(\tilde{u}_{f}\right) \leq 0 \\
& \tilde{u}_{\min } \leq \tilde{u}_{s}+\tilde{u}_{f} \leq \tilde{u}_{\max }
\end{array}
$$

with

$$
\begin{aligned}
J_{s}\left(\tilde{u}_{s}\right)= & \int_{0}^{1} x^{2} p_{s}(x) \frac{\partial}{\partial x}\left(a_{2}(x) \tilde{T}_{s}+a_{3}(x) \frac{\partial \tilde{T}_{s}}{\partial x}\right) \tilde{z} d x \\
& +\int_{0}^{1} x^{2} p_{s}(x) \frac{\partial}{\partial x}\left(a_{5}(x) \tilde{j}_{a u x}\left(\tilde{u}_{s}, x\right)\right) \tilde{z} d x \\
J_{f}\left(\tilde{u}_{f}\right) & =\int_{0}^{1} x^{2} p_{f}(x) b_{5}(x) \tilde{Q}_{a u x, f}\left(\tilde{u}_{f}, x\right) \tilde{T}_{f} d x
\end{aligned}
$$

The weights $\omega_{1}, \omega_{2}>0$ are the tuning parameters of the multi-objective optimization problem, and $\tilde{T}_{s}$ is found as the solution of (22) at each time instant using $\tilde{z}$ and $\tilde{u}_{s}$.

\section{RESULTS}
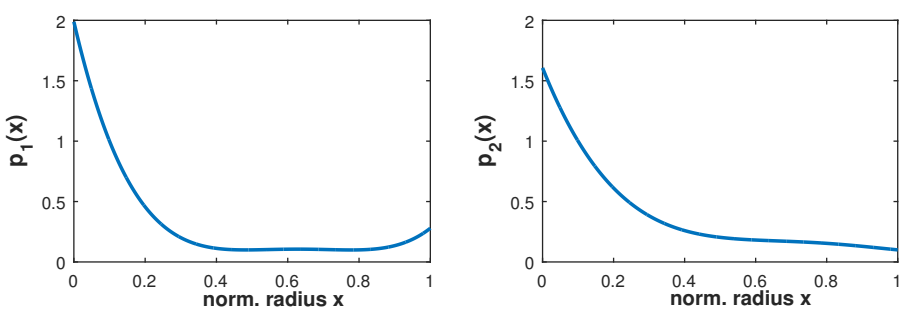

Fig. 3: Numerical solution of $p_{1}(x)$ and $p_{2}(x)$ for TCV

The two control strategies presented in the previous sections are evaluated using the nonlinear tokamak simulator RAPTOR. While real tokamak experiments for feedback control purposes are particularly difficult to obtain, RAPTOR provides a valid alternative as it includes the main physical properties of the plasma at the time scales of our study and has been
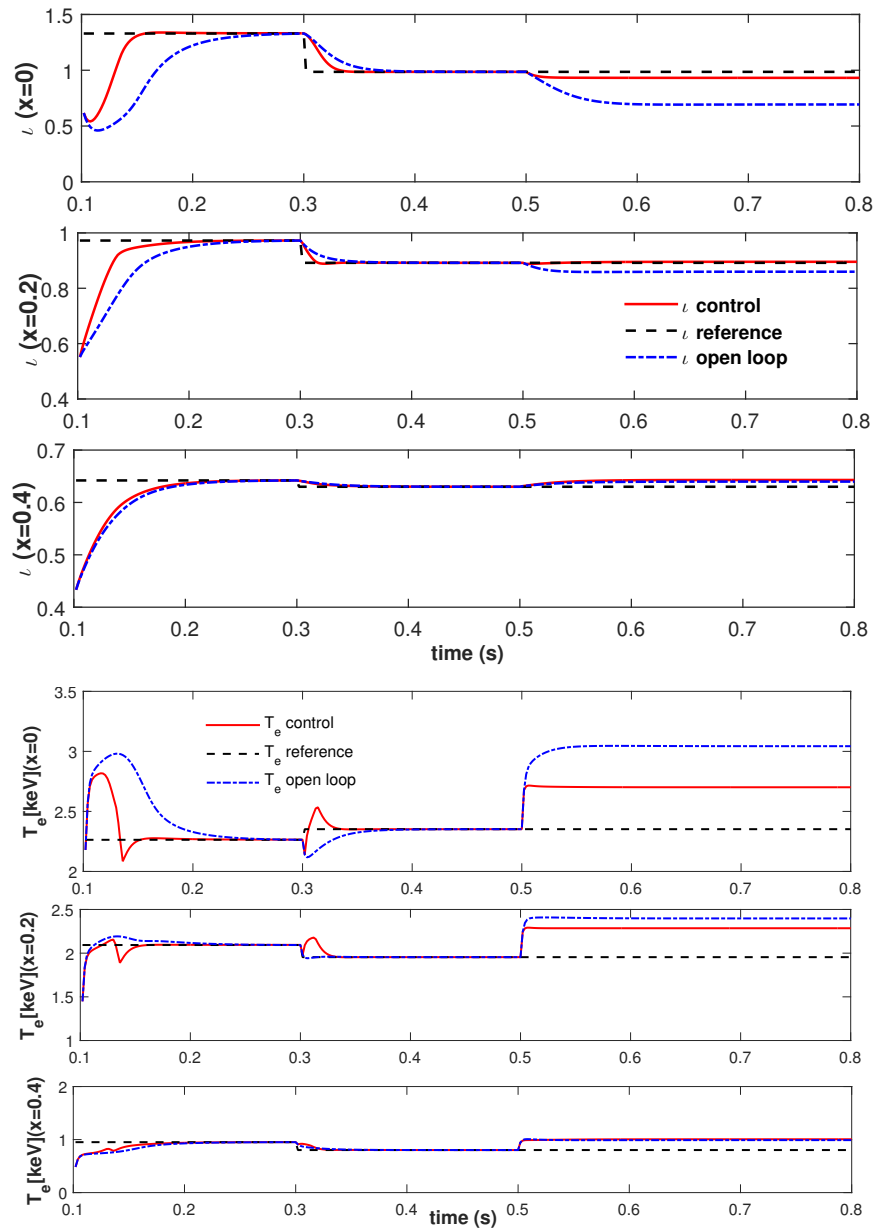

Fig. 5: Evolution of $\iota$ (top) and $T_{e}$ (bottom) at different plasma radii for the TCV simulation. The reference profile is modified at $t=0.3 \mathrm{~s}$ and a disturbance (extra source) is added at $t=$ $0.5 \mathrm{~s}$.
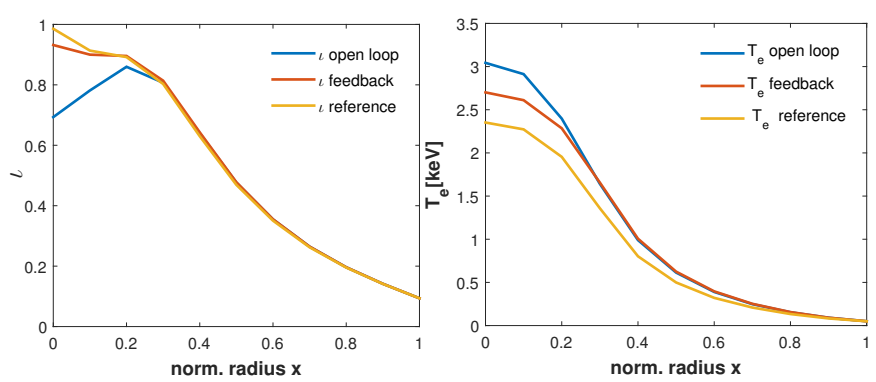

Fig. 6: Comparison of the final profiles of $\iota$ and $T_{e}$ obtained in open and closed loop for the TCV simulation.

successfully compared with experimental measurements on numerous test cases. Our first control approach (coupled case) is first evaluated on the small tokamak TCV $(\epsilon \approx 0.07)$ and then on the largest one ITER $(\epsilon \approx 0.01)$. It is compared on ITER with our second control approach, emphasizing the interest for considering the different time scales separately for large tokamaks. 


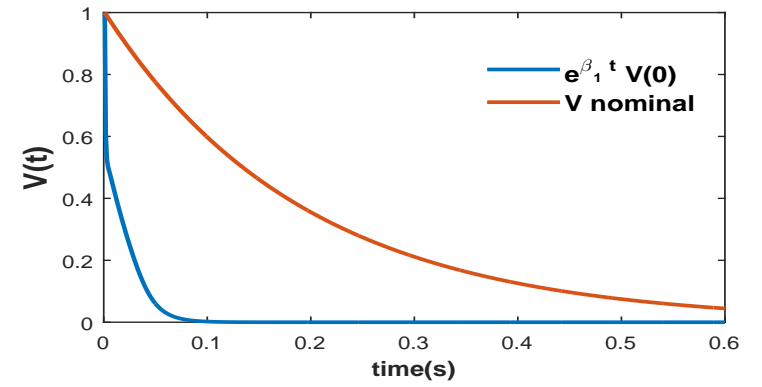

(a) Comparing the convergence rate of the nominal system with the calculated exponential convergence rate.

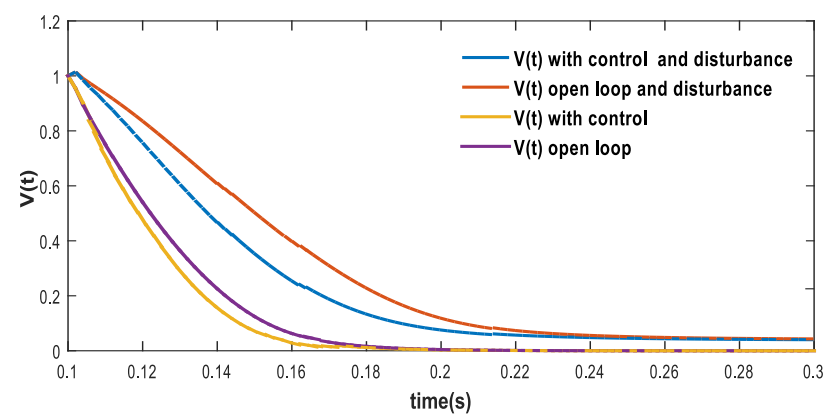

(b) Comparing the open-loop and the closed-loop convergence rates.

Fig. 4: Time-evolution of the normalized Lyapunov function in TCV simulations.

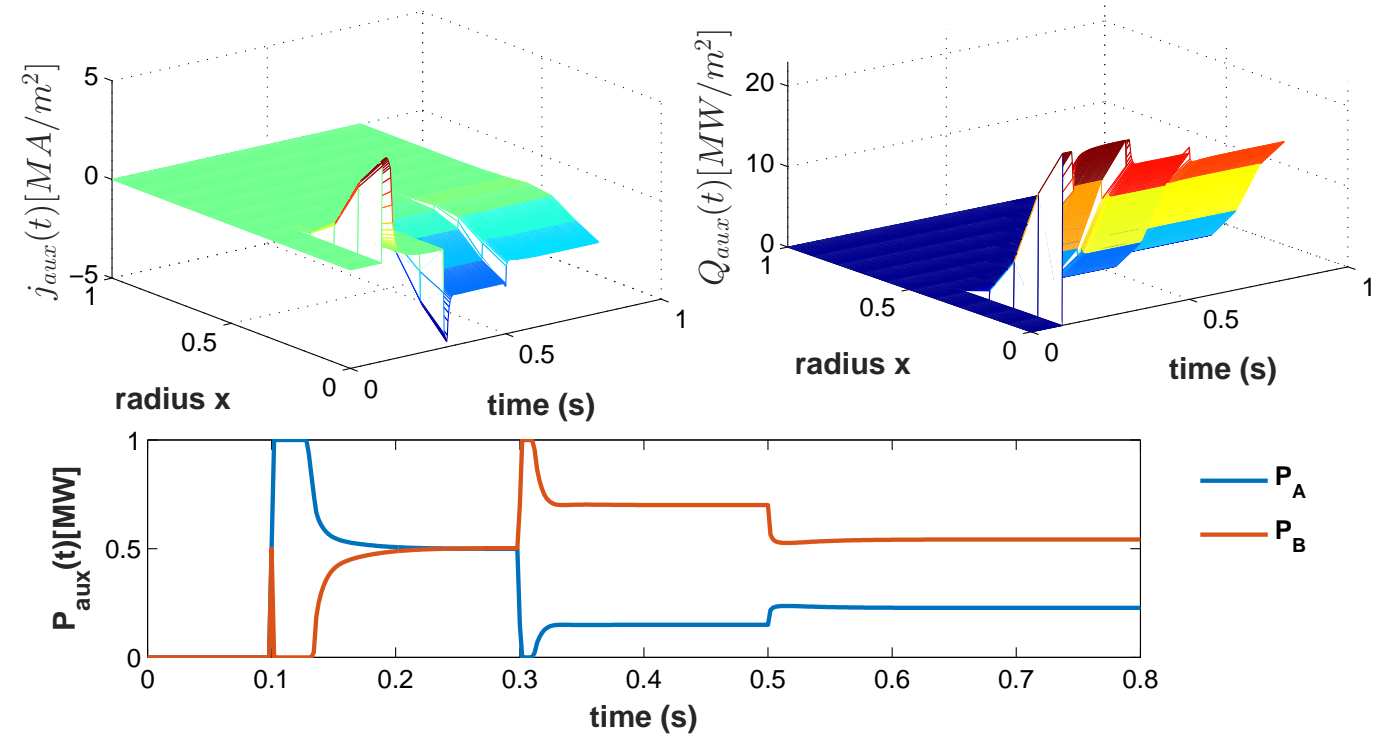

Fig. 7: Evolution of $j_{a u x}(x, t)\left[A / m^{2}\right]$ (top left), $Q_{a u x}(x, t)\left[W / m^{3}\right]$ (top right) and $P_{a u x}(t)$ (below) during the feedback control of TCV.

\section{A. TCV control}

The strategy presented in Section IV is tested on the TCV configuration. The feedback control is calculated solving the optimization problem given in (40). In this configuration, the flat-top plasma current is set to a constant $I_{p}=120 \mathrm{kA}$, while the EC antennas are used as control inputs. The control inputs are those available in the real TCV experiment and constituted by two EC heating and current drive antennas. The positions of the antennas set one on-axis current deposition with power $P_{e c 1}$ and an off-axis one with power $P_{e c 2}$. The deposition width is $\omega_{\text {dep }}=0.35$ and the location of its peak is $x_{d e p}=0$ for both clusters. The configuration of the reference values of $\bar{j}_{\text {aux }}$ and $\bar{Q}_{a u x}$ is shown in Fig. 1. The linearized model is obtained by extracting the parameters of the model corresponding to a stationary state when a constant value of the powers of the antennas is applied with $P_{e c 1}=P_{e c 2}=500 \mathrm{~kW}$.

Using these plasma parameters, the Lyapunov function of the system is calculated using the method presented in Section IV-B and the polynomials of the Lyapunov function were obtained as $5^{\text {th }}$ order Legendre polynomials, presented in Fig. 3. The solver finds the maximum value of $\alpha_{1}=0.03$, which provides some robustness margin. In Fig. 4a the evolution of the nominal system (zero inputs) is compared with the calculated exponential convergence rate $e^{-\beta_{1} t} \mathcal{V}(0)$. We can see that the inequality is satisfied and is relatively conservative, as a larger value of the convergence rate could be found. The calculation of the Lyapunov function proves its existence and the stability of the dynamics around the linearization profiles.

To test the control approach, several reference profiles are extracted, with open-loop simulation, using different fixed values of the powers $\bar{P}_{e c 1}$ and $\bar{P}_{e c 2}$. The reference profiles are used as reference trajectories for the tracking control and the corresponding powers vector $\bar{u}$ are included as a feedforward input, e.g. $u=\bar{u}+\tilde{u}$. In real tokamak experiment, the calculation of the feed-forward stationary values should be done using online numerical methods to solve the static plasma equations, for example as the one proposed in [18].

Saturations are set on the actuators and the power inputs are limited within the range of 0 to $1 \mathrm{MW}$. The reference profile is modified at $t=0.3 \mathrm{~s}$ to test the performance when the values of the nonlinear plasma parameters are different 
from the values used for linearization (modeling errors). At $0.5 \mathrm{~s}$ a disturbance is introduced using a third EC source with $x_{d e p}=0.2$ and $\omega_{d e p}=0.35$, to illustrate the robustness of the controller with respect to deviations from the calculated equilibrium point. The simulation results for $\iota$ and $T_{e}$ tracking are presented in Fig. 5: the feedback controller starts at $0.1 \mathrm{~s}$ and the results are compared with the open-loop (feedforward only) response of the system. Comparing the open and closed loop responses, the feedback control clearly gives a faster convergence rate and manages to attenuate the offset induced by the disturbances. This is further emphasized on the final values of the $\iota$ and $T_{e}$ profiles in Fig. 6. The control inputs are presented in Fig. 7, showing the evolution of the current and heat depositions, and the related input powers. The input saturations during the transients are successfully handled by the controller.

The time-evolutions of the Lyapunov function for several cases are compared in Fig. 4b. In this figure, the convergence rates of the closed-loop and the open-loop responses are compared with and without disturbance. In the both cases, the closed-loop system converges faster to the reference point than the open-loop system. The convergence rate of the closed-loop system is set by tuning the value of $\alpha_{2}$. Increasing the value of this parameter decreases the settling time and the steady state error. However, if $\alpha_{2}$ is too large the system response begins to oscillate and may become unstable. An $\alpha_{2}$ too large also implies a large input usage and larger overshoot of the fast-varying electron temperature.

\section{B. ITER control}

Our control methods are evaluated and compared on a second tokamak by setting RAPTOR with a configuration based on the ITER machine in L-mode. Even though RAPTOR is not the most suitable simulator for ITER and the model is not as accurate as the one of TCV, this configuration is used to test the performance of the composite control in a large scale tokamak that has a larger difference in the time scales. In this configuration three EC antennas are used as actuators. The reference values of $\bar{j}_{a u x}$ and $\bar{Q}_{a u x}$ are presented in Fig. 2. The plasma current is set to a constant $I_{p}=7 \mathrm{MA}$ and a linearized model is obtained by extracting the parameters corresponding to a stationary state when constant values of the powers of the EC antennas are $P_{e c 1}=P_{e c 2}=P_{e c 3}=7 \mathrm{MW}$. The input powers are limited in the range of 0 to $10 \mathrm{MW}$. As for the TCV simulations, several reference profiles are obtained to test the controller performance. An additional EC antenna is introduced to test the robustness with respect to deviations from the calculated equilibrium point.

Since both control methods necessitate a choice concerning the relative importance of the magnetic flux control with respect to the electrons heat control, we consider two control cases: Case 1 emphasizes the convergence rate of $\tilde{z}$ while $\tilde{T}_{e}$ is the priority for Case 2. Both control methods are evaluated on each case.

We first implement the coupled control presented in Section IV. The convergence rate is set by the tuning parameter $\alpha_{1}$ and the nature of the response depends highly on the choice of
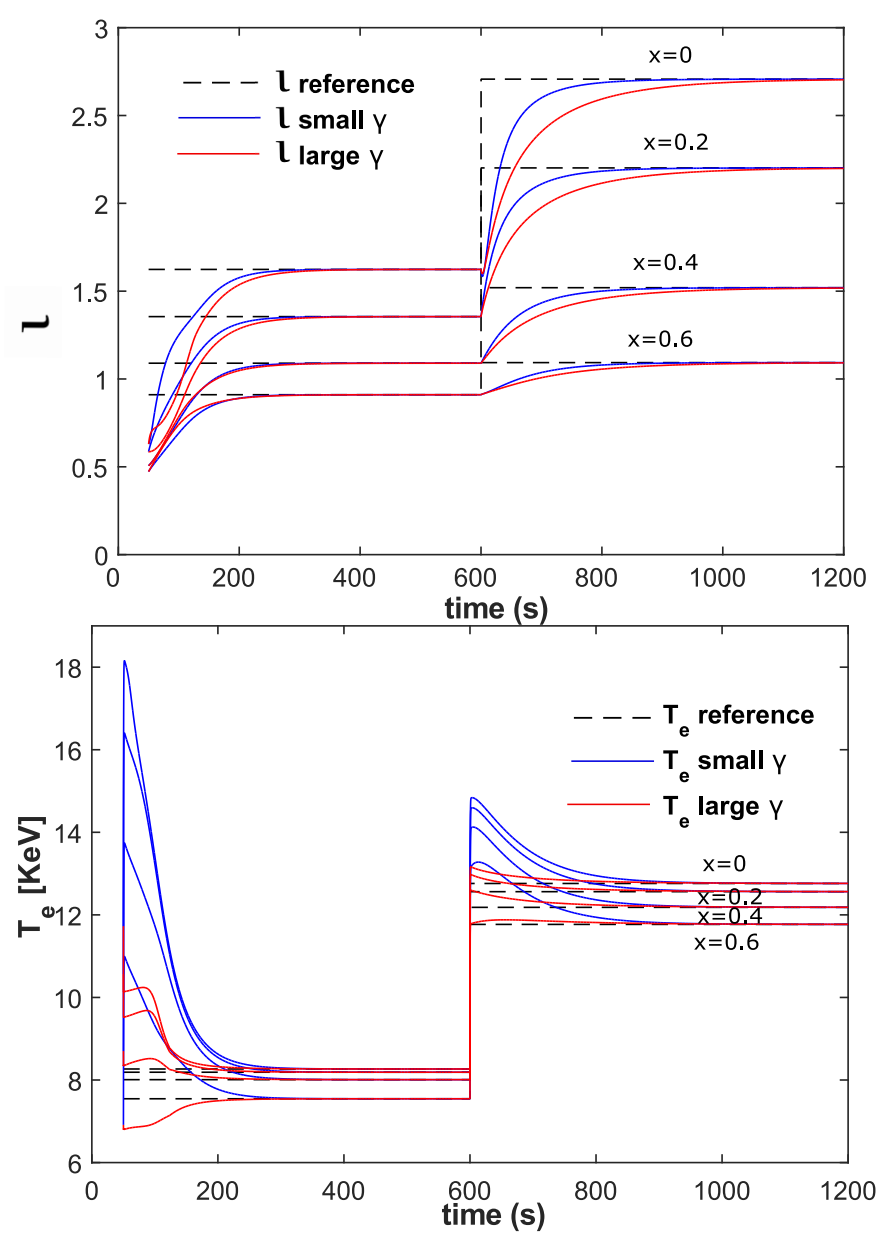

Fig. 8: Evolution $\iota$ (top) and $T_{e}$ (bottom) in the ITER simulation with the coupled controller and with convergence priority given to $\tilde{z}$ (Case 1 , small $\gamma$ ) or $\tilde{T}_{e}$ (Case 2, large $\gamma$ ).

the scaling parameter $\gamma$ (which multiplies $\tilde{T}_{e}$ in the Lyapunov function (14)). When $\gamma$ is low (Case 1), the control is more effective for the performance of the slow variable $\tilde{z}$ while the convergence rate of $T_{e}$ is difficult to tune (typically enduring high overshoots). When the value of $\gamma$ is high (Case 2), the performance of the convergence rate of $T_{e}$ can be tuned but the convergence rate of $\tilde{z}$ is free. To improve the convergence rate of $\tilde{z}$ we need in this case to increase the value of $\alpha_{1}$, which induces oscillations in the $T_{e}$ profile. The results from these simulations are presented in Fig. 8. While the convergence of $\iota$ and $T_{e}$ is achieved in both control cases, the disparity in the convergence rates of the two dynamics renders the relative tuning particularly difficult to achieve, motivating the system decoupling approach.

We now apply the composite control presented in Section V. The polynomials of the Lyapunov functions, presented in Fig. 10, are computed separately for the fast and the slow components. The maximum values of the convergence rate parameters are calculated numerically as $\alpha_{4}=0.01$ and $\alpha_{7}=9.3$. The difference between these two values was expected due to the difference in the time scales. The solution of the slow component of the temperature (22) is numerically calculated at each time instant and subtracted 

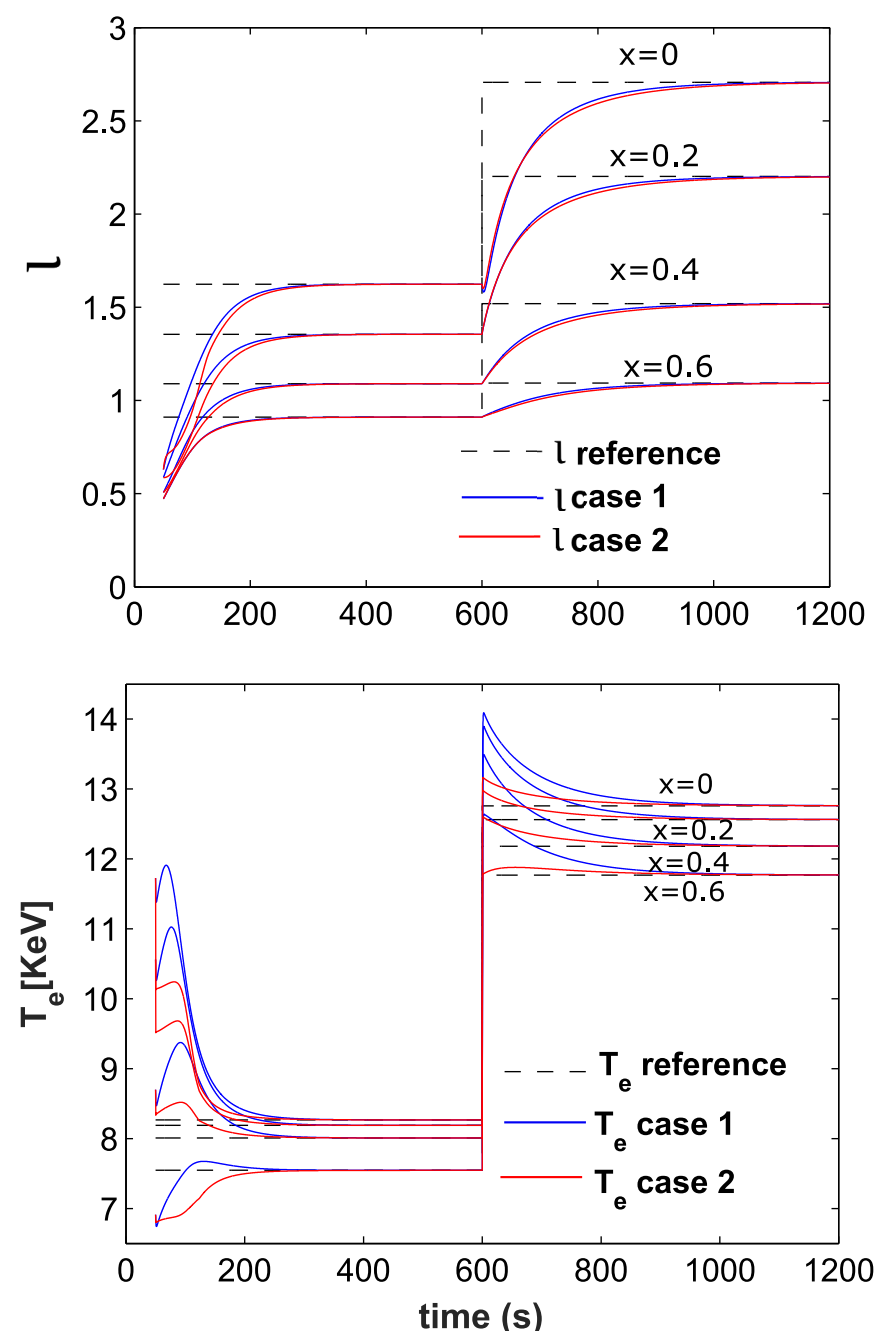

Fig. 9: Evolution of $T_{e}$ and $\iota$ in the ITER simulation with composite control for Case 1 and Case 2 .

from $\tilde{T}_{e}$ to estimate the fast component of the temperature $\tilde{T}_{f}$. The output parameters $\iota(x, t)$ and $T_{e}(x, t)$ at several locations are presented in Fig. 9 for the two control cases. For Case 1 a feedback control is applied only on the slow component of the system. The results from this simulation have similar performance as in the case when the method presented in Section IV is applied and low $\gamma$ is used. For Case 2 the composite control combines the feedback control of the fast and slow components. The effect of the feedback control on the boundary layer system can be observed on $T_{e}(x, t)$ : applying a control on the boundary layer system results in a reduced overshoot and a better convergence of the fast component at the cost of a slower convergence of the slow component. This behavior is also observed on the timeevolution of the slow and the fast Lyapunov functions starting from $t=600 \mathrm{~s}$ (when the reference profiles are changed) in Fig. 11. The controlled inputs for these simulations are presented in Fig. 13 (Case 1) and Fig. 12 (Case 2).

The tuning of the closed-loop performance with the composite control can be done by changing the values of the weighting parameters $\omega_{1,2}$ to obtain the desired balance between the two components. The convergence rate of the closed-loop system is selected by the choice of $\alpha_{4}$ and $\alpha_{6}$ for the performance of the slow and fast component, respectively. Increasing the value of $\alpha_{4}$ decreases the settling time and decreases the steady state error of the slow component, but increases the overshoot of the electron temperature. By increasing the value of $\alpha_{6}$ the convergence rate of the fast component is improved and the overshot of the electron temperature is decreased. If $\alpha_{6}$ is increased further, it leads to high oscillations of the fast varying component. Note also that when the two control functions are calculated separately we can apply different sampling periods for the two parameters, which improves the computational efficiency and the performance of the feedback control.
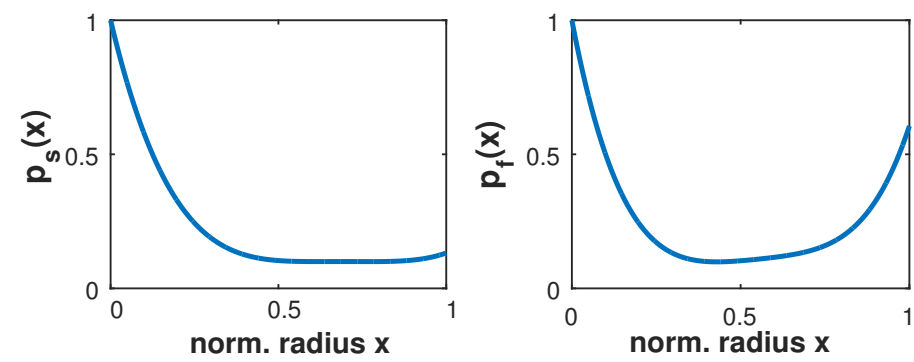

Fig. 10: Numerical solution of the composite control polynomials $p_{s}$ and $p_{f}$ for ITER.

\section{CONCLUSIONS AND FUTURE WORK}

Integrated feedback control algorithms for coupled current and electron temperature profiles in tokamaks are developed and tested in this work. The plasma states are modeled as two coupled inhomogeneous 1D diffusion PDEs and a controloriented model is proposed using linearized simple models for the controlled plasma parameters. The control design is based on the infinite dimensional settings by using control Lyapunov functions. Our first control method is based on the coupled dynamics and on a single Lyapunov function. The different time scales of the two states motivated a second control strategy designed by decoupling the two time scales of the system using singular perturbation theory. Both controllers are evaluated using the nonlinear RAPTOR tokamak plasma simulator, parametrized for the TCV and ITER tokamak machines. The different times scales in the two machines permits to compare the two control strategies. In the future, our control strategies can be used in a real tokamak machine using the state observer for the plasma profiles developed in [35]. A future perspective is also to consider the ohmic heating sources in the control design, as a boundary input.

\section{APPENDIX A}

\section{SIMPLIFIED MODELS OF THE PLASMA PARAMETERS}

The parallel resistivity of the plasma scales inversely with the electron temperature. In this work we are using the neoclassical resistivity model as in [36]:

$$
\eta_{\|}=c_{\text {neo }}(\rho) \eta_{\text {Spitz }}
$$



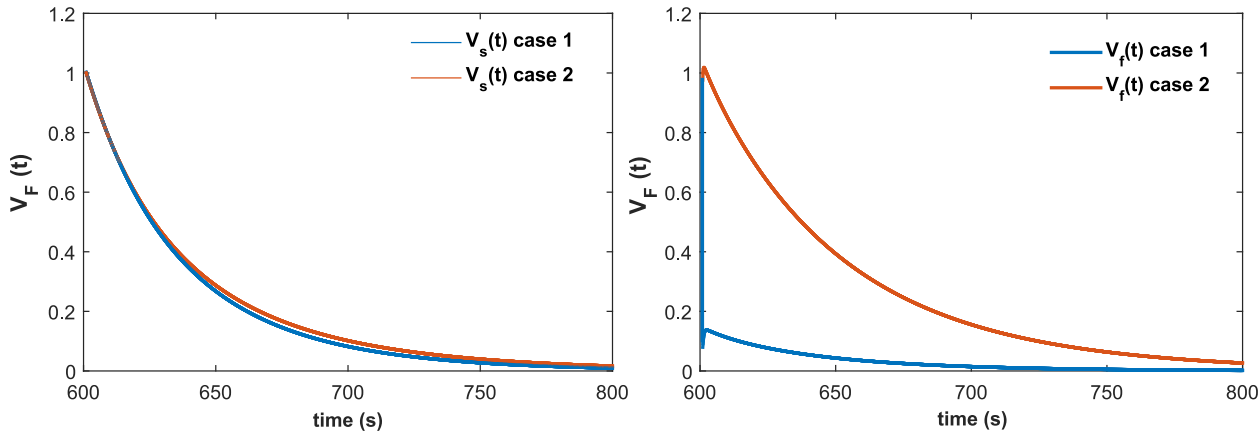

Fig. 11: Time-evolution of the two normalized Lyapunov functions of the composite control $\left(V_{s}\right.$ and $\left.V_{f}\right)$ for the ITER simulation.

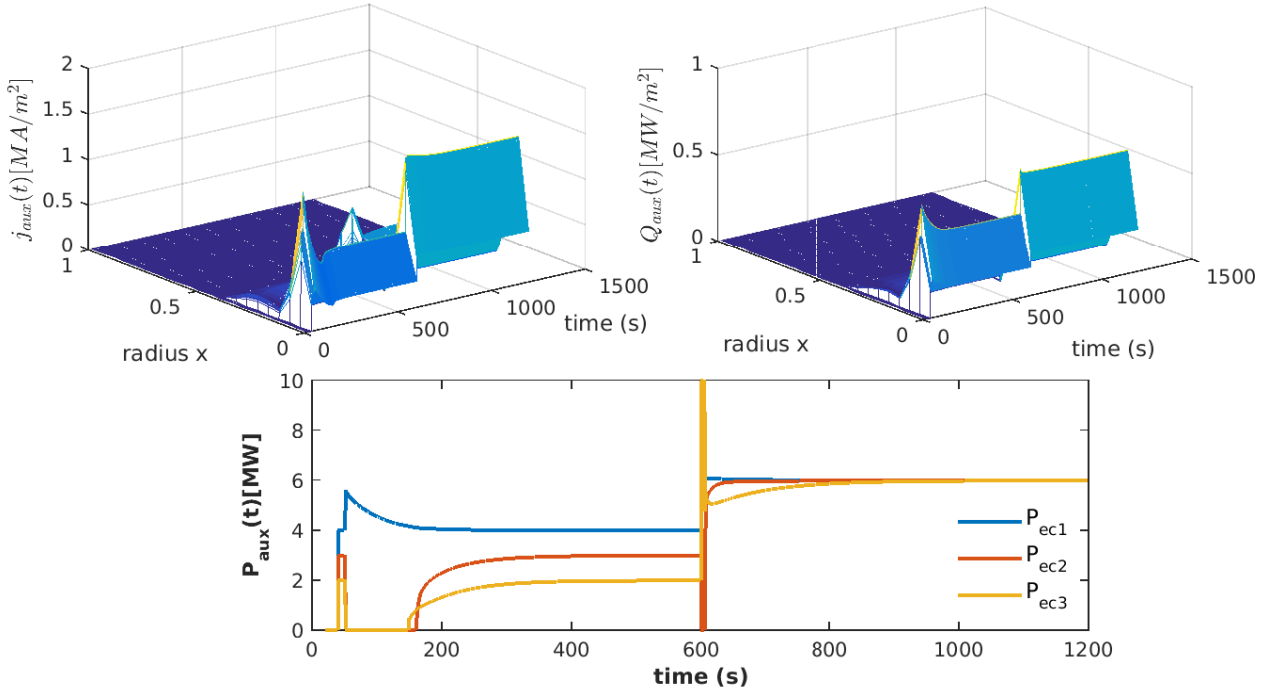

Fig. 12: Evolution of the input profiles $\tilde{j}_{a u x}(x, t)\left[A / m^{2}\right]$ (top left) and $\tilde{Q}_{a u x}(x, t)\left[W / m^{3}\right]$ (top right), and input powers $P_{\text {aux }}(t)$ (below) applied to the ITER configuration with composite control for Case 1.

where $c_{n e o}(\rho)$ is the neoclassical correction, and the Spitzer conductivity $\eta_{\text {Spitz }}$ is given by:

$$
\eta_{\text {Spitz }}=\frac{k_{s p}(\rho) Z_{\text {eff }}}{T_{e}^{3 / 2}}
$$

where $k_{s p}(\rho)$ is the spatial profile and $Z_{e f f}$ is the constant effective value of the plasma charge.

The bootstrap current is a self-generated current due to collisions between trapped particles and passing particles. The model of the bootstrap current density is simplified as in [9]:

$$
\begin{aligned}
j_{b s} & =\frac{k_{b s}(x)}{\partial \Psi / \partial x}\left(\mathcal{L}_{31}(x) \frac{\partial n_{e}}{\partial x} T_{e}\right. \\
& \left.+\left(\mathcal{L}_{31}(x)+R_{p e} \mathcal{L}_{32}(x)+\left(1-R_{p e}\right) \mathcal{L}_{34}(x)\right) \frac{\partial T_{e}}{\partial x} n_{e}\right)
\end{aligned}
$$

where $k_{b s}(x), \mathcal{L}_{31}(x), \mathcal{L}_{32}(x), \mathcal{L}_{34}(x)$ depend on magnetic configuration of the plasma equilibrium and $R_{p e}$ is the ratio between electron and total pressure.

The heat diffusion coefficient in the electrons temperature equation is not well known and there is no consensus about the mathematical formulation: only some empirical models are developed for this coefficient. For the control model in this work a simple empirical local transport model of the Bohm type is used, given as in [37]:

$$
\chi_{e}=2.510^{-4} \frac{T_{e}}{B_{0}} \frac{\left|\nabla p_{e}\right|}{p_{e}} q^{2} \propto 2.510^{-4} \frac{\left|\nabla n_{e} T_{e}\right|}{B_{0} n_{e}} q^{2}
$$

where $p_{e}=n_{e} T_{e}$ is the electron pressure. The ohmic effect power density $Q_{O H}$ comes from the heating caused by the induced current as:

$$
Q_{O H}=\frac{\eta_{\|}}{\mu_{0}^{2} R_{0}^{2} a^{3} x^{2}}\left(\frac{\partial}{\partial x}(x z)\right)^{2}
$$

The electron-ion heat exchange can be written as $Q_{e i}=$ $n_{e} \nu_{e q}\left(T_{e}-T_{i}\right)$, where $\nu_{e q}$ is the neoclassical equipartition rate given in [30].

For the ion temperature, a simplified model is used, where it is directly related to $T_{e}$ by $T_{i}=f_{T i}(x) T_{e}$, where $f_{T i}(x)$ is a function chosen to best match the simulation results as in [8]. The electron-ion heat exchange can then be written in terms of $T_{e}$ as:

$$
\begin{aligned}
Q_{e i} & =n_{e} \nu_{e q}\left(T_{e}-T_{i}\right) \\
& =n_{e} \nu_{e q}\left(1-f_{T i}(x)\right) T_{e}=f_{e i}(x) T_{e}
\end{aligned}
$$



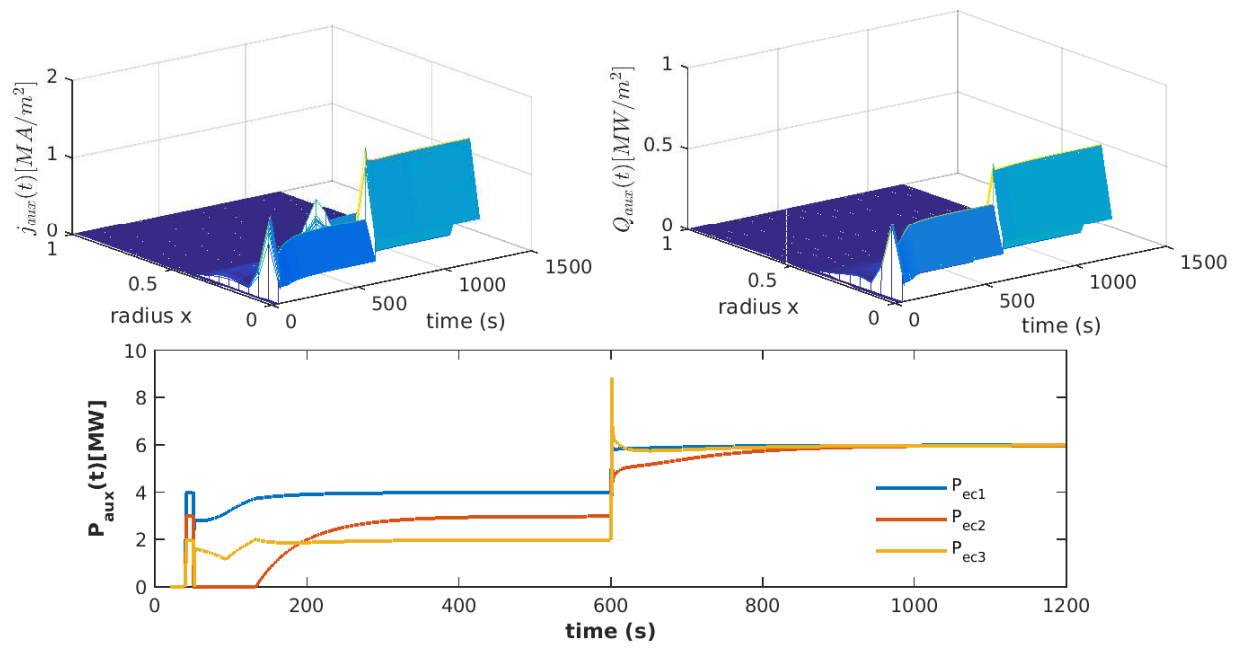

Fig. 13: Evolution of the input profiles $\tilde{j}_{a u x}(x, t)\left[A / m^{2}\right]$ (top left) and $\tilde{Q}_{a u x}(x, t)\left[W / m^{3}\right]$ (top right), and input powers $P_{\text {aux }}(t)$ (below) applied to the ITER configuration with composite control for Case 2.

The radiation loss power density $Q_{\text {rad }}[1]$ is calculated as:

$$
Q_{\text {rad }}=f_{\text {rad }}(x) T_{e}^{1 / 2}
$$

where $f_{\text {rad }}=k_{\text {bem }} n_{e}^{2} Z_{\text {eff }}$ and $k_{\text {bem }}=5.35 \times$ $10^{5} \mathrm{Wm}^{3} /(\mathrm{keV})^{1 / 2}$ is the Bremsstrahlung radiation coefficient.

\section{APPENDIX B}

PROOF OF THEOREM 1

Proof. The time derivative of the Lyapunov function is:

$$
\begin{aligned}
\dot{V} & =\int_{0}^{1} x^{2} p_{1}(x) \frac{\partial \tilde{z}}{\partial t} \tilde{z} d x+\gamma \int_{0}^{1} x^{2} p_{2}(x) \frac{\partial \tilde{T}_{e}}{\partial t} \tilde{T}_{e} d x \\
& =\dot{V}_{1,1}+\dot{V}_{1,2}+\dot{V}_{2,1}+\dot{V}_{2,2}+\dot{V}_{2,3}+\dot{V}_{2,4}
\end{aligned}
$$

where:

$$
\begin{gathered}
\dot{V}_{1,1}=\int_{0}^{1} x^{2} p_{1}(x) \frac{\partial}{\partial x}\left(\frac{a_{1}(x)}{x} \frac{\partial}{\partial x}(x \tilde{z})\right) \tilde{z} d x \\
\dot{V}_{1,2}=\int_{0}^{1} x^{2} p_{1}(x) \frac{\partial}{\partial x}\left(a_{2}(x) \tilde{T}_{e}+a_{3}(x) \frac{\partial \tilde{T}_{e}}{\partial x}+a_{4}(x) \tilde{z}\right) \tilde{z} d x \\
\dot{V}_{2,1}=\frac{1}{\varepsilon} \int_{0}^{1} x p_{2}(x) \frac{1}{x} \frac{\partial}{\partial x}\left(x b_{1}(x) \frac{\partial \tilde{T}_{e}}{\partial x}\right) \tilde{T}_{e} d x \\
\dot{V}_{2,2}=-\frac{1}{\varepsilon} \int_{0}^{1} x^{2} p_{2}(x) b_{2}(x) \tilde{T}_{e}^{2} \\
\dot{V}_{2,3}=\frac{1}{\varepsilon} \int_{0}^{1} x p_{2}(x) \frac{\partial}{\partial x}\left(x b_{3}(x) \tilde{z}\right) \tilde{T}_{e} \\
\dot{V}_{2,4}=\frac{1}{\varepsilon} \int_{0}^{1} x p_{2}(x) b_{4}(x)\left(\frac{\partial}{\partial x}(x \tilde{z})\right) \tilde{T}_{e} d x \\
\dot{V}_{2,5}=\frac{1}{\varepsilon} \int_{0}^{1} x^{2} p_{2}(x) b_{5}(x) \tilde{Q}_{a u x}(\tilde{u}, x, t) \tilde{T}_{e} d x
\end{gathered}
$$

Integrating $\dot{V}_{1,1}$ by parts and considering the boundary conditions (12) we get:

$$
\begin{aligned}
\dot{V}_{1,1}= & \left.x p_{1}(x) \tilde{z} a_{1}(x) \frac{\partial}{\partial x}(x \tilde{z})\right|_{0} ^{1} \\
& -\int_{0}^{1} x^{2} p_{1}(x) a_{1}(x)\left(\frac{\partial \tilde{z}}{\partial x}\right)^{2} d x \\
& -\int_{0}^{1}\left(2 p_{1}(x)+x p_{1}^{\prime}(x)\right) a_{1}(x) \tilde{z}^{2} d x \\
& -\int_{0}^{1}\left(x^{2} p_{1}^{\prime}(x)+3 x p_{1}(x)\right) a_{1}(x) \tilde{z} \frac{\partial \tilde{z}}{\partial x} d x
\end{aligned}
$$

Integrating again by parts some terms in $\dot{V}_{1,1}$, we get:

$$
\begin{aligned}
\dot{V}_{1,1}= & -\left.\frac{1}{2} a_{1}(x)\left(x^{2} p_{1}^{\prime}(x)+3 x p_{1}(x)\right) \tilde{z}^{2}\right|_{0} ^{1} \\
& -\int_{0}^{1} x^{2} p_{1}(x) a_{1}(x)\left(\frac{\partial \tilde{z}}{\partial x}\right)^{2} d x \\
& +\frac{1}{2} \int_{0}^{1}\left(3 x p_{1}^{\prime}(x)+x^{2} p_{1}^{\prime \prime}(x)-p_{1}(x)\right) a_{1}(x) \tilde{z}^{2} d x \\
& +\frac{1}{2} \int_{0}^{1} a_{1}^{\prime}(x)\left(x^{2} p_{1}^{\prime}(x)+3 x p_{1}(x)\right) \tilde{z}^{2} d x
\end{aligned}
$$

Integrating $\dot{V}_{1,2}$ and $\dot{V}_{2,1}$ by parts and considering the boundary conditions (12) we get:

$$
\begin{aligned}
\dot{V}_{1,2}= & -\int_{0}^{1}\left(2 x p_{1}(x)+x^{2} p_{1}^{\prime}(x)\right) a_{2}(x) \tilde{z} \tilde{T}_{e} d x \\
& +\int_{0}^{1} x^{2} p_{1}(x) a_{2}(x) \frac{\partial \tilde{z}}{\partial x} \tilde{T}_{e} d x \\
& +\int_{0}^{1} x^{2} p_{1}(x) a_{3}(x) \frac{\partial \tilde{z}}{\partial x} \frac{\partial \tilde{T}_{e}}{\partial x} d x \\
& -\int_{0}^{1}\left(2 x p_{1}(x)+x^{2} p_{1}^{\prime}(x)\right) a_{3}(x) \tilde{z} \frac{\partial \tilde{T}_{e}}{\partial x} d x \\
& -\int_{0}^{1}\left(2 x p_{1}(x)+x^{2} p_{1}^{\prime}(x)\right) \tilde{z}^{2} a_{4}(x) \tilde{z} d x \\
& +x^{2} p_{1}(x) a_{4}(x) \frac{\partial \tilde{z}}{\partial x} \tilde{z} d x
\end{aligned}
$$


and

$$
\begin{aligned}
\dot{V}_{2,1}= & \left.\frac{1}{\varepsilon} x^{2} p_{2}(x) b_{1}(x) \tilde{T}_{e} \frac{\partial \tilde{T}_{e}}{\partial x}\right|_{0} ^{1} \\
& -\frac{1}{\varepsilon} \int_{0}^{1}\left(x p_{2}(x)+x^{2} p_{2}^{\prime}(x)\right) b_{1}(x) \tilde{T}_{e} \frac{\partial \tilde{T}_{e}}{\partial x} d x \\
& -\frac{1}{\varepsilon} \int_{0}^{1} x^{2} p_{2}(x) b_{1}(x)\left(\frac{\partial \tilde{T}_{e}}{\partial x}\right)^{2} d x \\
& =\frac{1}{2 \varepsilon} \int_{0}^{1} b_{1}(x)\left(\left(x^{2} p_{2}^{\prime \prime}(x)+2 x p_{2}^{\prime}(x)+p_{2}(x)\right) \tilde{T}_{e}^{2} d x\right. \\
& +\frac{1}{2 \varepsilon} \int_{0}^{1} b_{1}^{\prime}(x)\left(x^{2} p_{2}^{\prime}(x)+x p_{2}(x)\right) \tilde{T}_{e}^{2} d x \\
& -\frac{1}{\varepsilon} \int_{0}^{1} x^{2} p_{2}(x) b_{1}(x)\left(\frac{\partial \tilde{T}_{e}}{\partial x}\right)^{2} d x \\
& -\left.\frac{1}{\varepsilon}\left(p_{2}(x)+x p_{2}^{\prime}(x)\right) b_{1}(x) \tilde{T}_{e}^{2}\right|_{0} ^{1}
\end{aligned}
$$

We assume that $\tilde{T}_{e, \text { edge }}$ is very small compared to the temperature in the center of the plasma and we can consider it as zero in the analysis.

Integrating $\dot{V}_{2,3}$ and $\dot{V}_{2,4}$ by parts and considering the boundary conditions (12) we get:

$$
\begin{aligned}
\dot{V}_{2,3}= & -\frac{1}{\varepsilon} \int_{0}^{1} x b_{3}(x)\left(x p_{2}^{\prime}(x)+p_{2}(x)\right) \tilde{z} \tilde{T}_{e} d x \\
& -\frac{1}{\varepsilon} \int_{0}^{1} x^{2} b_{3}(x) p_{2}(x) \frac{\partial \tilde{z}}{\partial x} \tilde{T}_{e} d x
\end{aligned}
$$

and

$$
\begin{aligned}
\dot{V}_{2,4}= & \frac{1}{\varepsilon} \int_{0}^{1} x p_{2}(x) b_{4}(x) \tilde{z} \tilde{T}_{e} d x \\
& +\frac{1}{\varepsilon} \int_{0}^{1} x^{2} p_{2}(x) b_{4}(x) \frac{\partial \tilde{z}}{\partial x} \tilde{T}_{e} d x
\end{aligned}
$$

The derivative of the Lyapunov function combining (49)-
(52) can be written as:

$$
\begin{aligned}
\dot{V} & =\int_{0}^{1}\left[\begin{array}{c}
\tilde{z} \\
\tilde{T}_{e} \\
\frac{\partial \tilde{z}}{\partial x} \\
\frac{\partial \tilde{T}_{e}}{\partial x}
\end{array}\right]^{T} A_{1}(x)\left[\begin{array}{c}
\tilde{z} \\
\tilde{T}_{e} \\
\frac{\partial \tilde{z}}{\partial x} \\
\frac{\partial \tilde{T}_{e}}{\partial x}
\end{array}\right] d x \\
& +\int_{0}^{1} x^{2} p_{1}(x) \frac{\partial}{\partial x}\left(a_{4}(x) \tilde{j}_{a u x}(\tilde{u}, x, t)\right) \tilde{z} d x \\
& +\gamma \int_{0}^{1} \frac{x^{2} p_{2}(x)}{\varepsilon} b_{5}(x) \tilde{Q}_{a u x}(\tilde{u}, x, t) \tilde{T}_{e} d x
\end{aligned}
$$

where the matrix $A_{1}(X)$ is given in (16).

Combining the inequality (15) and (53) implies (19).

\section{APPENDIX C}

PROOF OF THEOREM 2

Proof. The time derivative of the Lyapunov function is:

$$
\begin{aligned}
\dot{V}_{s}= & \frac{1}{2} \int_{0}^{1} x^{2} p_{s}(x) \frac{\partial \tilde{z}}{\partial t} \tilde{z} d x \\
& =\int_{0}^{1} x^{2} p_{s}(x) \frac{\partial}{\partial x}\left(a_{1}(x) \frac{\partial}{\partial x}(x \tilde{z})\right) \tilde{z} d x \\
& +\int_{0}^{1} x^{2} p_{s}(x) \frac{\partial}{\partial x}\left(a_{4}(x) \tilde{z}\right) \tilde{z} d x \\
& +\int_{0}^{1} x^{2} p_{s}(x) \frac{\partial}{\partial x}\left(a_{2}(x) \tilde{T}_{s}+a_{3}(x) \frac{\partial \tilde{T}_{s}}{\partial x}\right) \tilde{z} d x \\
& +\int_{0}^{1} x^{2} p_{s}(x) \frac{\partial}{\partial x}\left(a_{5}(x) \tilde{j}_{a u x}\left(\tilde{u}_{s}, x, t\right)\right) \tilde{z} d x
\end{aligned}
$$

Integrating by parts and considering the boundary conditions (27), we get the following inequality:

$$
\begin{aligned}
\dot{V}_{s} \leq & \frac{1}{2} \int_{0}^{1}\left(3 x p_{s}^{\prime}(x)+x^{2} p_{s}^{\prime \prime}(x)-p_{s}(x)\right) a_{1}(x) \tilde{z}^{2} d x \\
& +\frac{1}{2} \int_{0}^{1} a_{1}^{\prime}(x)\left(x^{2} p_{s}^{\prime}(x)+3 x p_{s}(x)\right) \tilde{z}^{2} d x \\
& +\int_{0}^{1} a_{4}(x)\left(x p_{s}(x)+\frac{1}{2} x^{2} p_{1}^{\prime}(x)\right) \tilde{z}^{2} d x \\
& -\int_{0}^{1} \frac{1}{2} x^{2} a_{4}^{\prime}(x) p_{1}(x) \tilde{z}^{2} d x \\
& +\int_{0}^{1} x^{2} p_{s}(x) \frac{\partial}{\partial x}\left(a_{2}(x) \tilde{T}_{s}+a_{3}(x) \frac{\partial \tilde{T}_{s}}{\partial x}\right) \tilde{z} d x \\
& +\int_{0}^{1} x^{2} p_{s}(x) \frac{\partial}{\partial x}\left(a_{5}(x) \tilde{j}_{a u x}\left(\tilde{u}_{s}, x, t\right)\right) \tilde{z} d x
\end{aligned}
$$

$$
\begin{aligned}
& a_{1}(x)=\frac{\bar{\eta}_{\|}}{\mu_{0} a^{2}}, a_{2}(x)=\frac{1}{\mu_{0} a^{2}} \frac{\partial \bar{\eta}_{\|}}{\partial \bar{T}_{e}} \frac{\partial}{\partial x}(x(\bar{z}))+\frac{\partial \bar{\eta}}{\partial \bar{T}_{e}} R_{0} \bar{j}_{n i}+\frac{\partial \bar{j}_{a u x}}{\partial \bar{T}_{e}} \bar{\eta} R_{0}+\bar{\eta}_{\|} R_{0} \frac{k_{b s}(x)}{\bar{z}}\left(\mathcal{L}_{31}(x) \frac{\partial n_{e}}{\partial x}\right) \\
& a_{3}(x)=\bar{\eta}_{||} R_{0} n_{e} \frac{k_{b s}(x)}{\bar{z}}\left(\mathcal{L}_{31}(x)+R_{p e} \mathcal{L}_{32}(x)+\left(1-R_{p e}\right) \mathcal{L}_{34}(x)\right) \\
& a_{4}(x)=-\bar{\eta}_{||} R_{0} \frac{k_{b s}(x)}{\bar{z}^{2}}\left(\mathcal{L}_{31}(x) \frac{\partial n_{e}}{\partial x} \bar{T}_{e}+\left(\mathcal{L}_{31}(x)+R_{p e} \mathcal{L}_{32}(x)+\left(1-R_{p e}\right) \mathcal{L}_{34}(x)\right) \frac{\partial \bar{T}_{e}}{\partial x} n_{e}\right) \\
& a_{5}(x)=\bar{\eta}_{\|} R_{0}, b_{1}(x)=\frac{2 \varepsilon}{3 a^{2}}\left(\bar{\chi}_{e}+\frac{\partial \bar{T}_{e}}{\partial x} \frac{\partial \bar{\chi}_{e}}{\partial\left(\nabla \bar{T}_{e}\right)}\right), b_{2}(x)=-\frac{2 \varepsilon}{3 n_{e}}\left(f_{e i}(x)+\frac{1}{2} f_{r a d}(x) \bar{T}_{e}^{-1 / 2}-\frac{4}{3} \frac{\partial \bar{\eta}_{\| \mid}}{n_{e} \mu_{0}^{2} R_{0}^{2} a^{3} x^{2}}\left(\frac{\partial}{\partial x}(x \bar{z})\right)^{2}\right) \\
& b_{3}(x)=\frac{\varepsilon}{a^{2}} \frac{\partial \bar{\chi}_{e}}{\partial \bar{z}} \frac{\partial \bar{T}_{e}}{\partial x}, b_{4}(x)=\frac{4 \varepsilon}{3} \frac{\bar{\eta}_{\|}}{n_{e} \mu_{0}^{2} R_{0}^{2} a^{3} x}\left(\frac{\partial}{\partial x}(x \bar{z})\right), b_{5}(x)=\frac{2 \varepsilon}{3 n_{e}}
\end{aligned}
$$


Combining the inequalities (29) and (54) implies (30).

\section{APPENDIX D \\ PROOF OF THEOREM 3}

Proof. The derivative of the Lyapunov function for the fast dynamics is:

$$
\begin{aligned}
\dot{V}_{f} & =\int_{0}^{1} x^{2} p_{f}(x) \frac{1}{x} \frac{\partial}{\partial x}\left(x b_{1}(x) \frac{\partial \tilde{T}_{f}}{\partial x}\right) \tilde{T}_{f} d x \\
& -\int_{0}^{1} x^{2} p_{f}(x) b_{2}(x) \tilde{T}_{f}^{2} d x \\
& +\int_{0}^{1} x^{2} p_{f}(x) b_{5}(x) \tilde{Q}_{a u x, f}\left(\tilde{u}_{f}, x, t\right) \tilde{T}_{f} d x
\end{aligned}
$$

Integrating by parts and considering the boundary conditions of the boundary layer system (25), we have:

$$
\begin{aligned}
\dot{V}_{f}= & \frac{1}{2} \int_{0}^{1} b_{1}(x)\left(\left(x^{2} p_{f}^{\prime \prime}(x)+2 x p_{f}^{\prime}(x)+p_{f}(x)\right) \tilde{T}_{f}^{2} d x\right. \\
& +\frac{1}{2} \int_{0}^{1} b_{1}^{\prime}(x)\left(x^{2} p_{f}^{\prime}(x)+x p_{f}(x)\right) \tilde{T}_{f}^{2} d x \\
& -\int_{0}^{1} x^{2} p_{f}(x) b_{1}(x)\left(\frac{\partial \tilde{T}_{f}}{\partial x}\right)^{2} d x \\
& -\int_{0}^{1} x^{2} p_{f}(x)\left(b_{2}(x)\right) \tilde{T}_{f}^{2} d x \\
& +\int_{0}^{1} x^{2} p_{f}(x) b_{5}(x) \tilde{Q}_{a u x, f}\left(\tilde{u}_{f}, x, t\right) \tilde{T}_{f} d x
\end{aligned}
$$

Combining the inequalities (33) and (56) implies (34).

\section{ACKNOWLEDGMENTS}

The authors would like to thank Federico Felici for his patience and invaluable help in the use of RAPTOR. This work has been partially supported by the ANR project TORID contract number ANR-12-BS03-0008.

\section{REFERENCES}

[1] J. Wesson, Tokamaks, $4^{\text {th }}$ ed., ser. International Series of Monographs on Physics. Oxford, UK: Oxford University Press, 2011, vol. 149.

[2] A. Pironti and M. Walker, "Fusion, tokamaks, and plasma control: an introduction and tutorial," IEEE Control System Magazine, vol. 25, no. 5, pp. 30-43, 2005.

[3] D. Moreau, D. Mazon, M. Ariola, G. De Tommasi, L. Laborde, F. Piccolo, F. Sartori, T. Tala, L. Zabeo, A. Boboc et al., "A two-timescale dynamic-model approach for magnetic and kinetic profile control in advanced tokamak scenarios on JET," Nuclear Fusion, vol. 48, no. 10, p. 106001, 2008.

[4] D. Moreau, M. L. Walker, J. R. Ferron, F. Liu, E. Schuster, J. E. Barton, M. D. Boyer, K. H. Burrell, S. Flanagan, P. Gohil et al., "Integrated magnetic and kinetic control of advanced tokamak plasmas on DIII-D based on data-driven models," Nuclear Fusion, vol. 53, no. 6, p. 063020 , 2013.

[5] D. Moreau, D. Mazon, M. Walker, J. Ferron, K. Burrell, S. Flanagan, P. Gohil, R. Groebner, A. Hyatt, R. La Haye et al., "Plasma models for real-time control of advanced tokamak scenarios," Nuclear Fusion, vol. 51, no. 6, p. 063009, 2011.

[6] B. Mavkov, E. Witrant, C. Prieur, and D. Moreau, "Multi-experiment state-space identification of coupled magnetic and kinetic parameters in tokamak plasmas," Control Engineering Practice, vol. 60, pp. 28-38, 2017.
[7] J. Blum, Numerical simulation and optimal control in plasma physics with applications to Tokamaks, ser. Modern Applied Mathematics. Wiley / Gauthier-Villars, 1989.

[8] E. Witrant, E. Joffrin, S. Brémond, G. Giruzzi, D. Mazon, O. Barana, and P. Moreau, "A control-oriented model of the current profile in tokamak plasma," Plasma Physics and Controlled Fusion, vol. 49, no. 7, p. 1075 , 2007.

[9] F. Felici, O. Sauter, S. Coda, B. Duval, T. Goodman, J. Moret, J. Paley, T. Team et al., "Real-time physics-model-based simulation of the current density profile in tokamak plasmas," Nuclear Fusion, vol. 51, no. 8, p. 083052, 2011.

[10] J. E. Barton, W. P. Wehner, E. Schuster, F. Felici, and O. Sauter, "Simultaneous closed-loop control of the current profile and the electron temperature profile in the TCV tokamak," in American Control Conference, Chicago, USA, 2015, pp. 3316-3321.

[11] M. van Berkel, H. J. Zwart, G. M. D. Hogeweij, G. Vandersteen, $\mathrm{H}$. van den Brand, M. R. de Baar, and the ASDEX Upgrade Team, "Estimation of the thermal diffusion coefficient in fusion plasmas taking frequency measurement uncertainties into account," Plasma Physics and Controlled Fusion, vol. 56, no. 10, p. 105004, 2014.

[12] S. Mechhoud, E. Witrant, L. Dugard, and D. Moreau, "Estimation of heat source term and thermal diffusion in tokamak plasmas using a Kalman filtering method in the early lumping approach," IEEE Transactions on Control Systems Technology, vol. 23, no. 2, pp. 449-463, 2015.

[13] D. Kim, A. Merle, O. Sauter, and T. Goodman, "Simple predictive electron transport models applied to sawtoothing plasmas," Plasma Physics and Controlled Fusion, vol. 58, no. 5, p. 055002, 2016.

[14] L. Laborde, D. Mazon, D. Moreau, A. Murari, R. Felton, L. Zabeo, R. Albanese, M. Ariola, J. Bucalossi, F. Crisanti et al., "A model-based technique for integrated real-time profile control in the JET tokamak," Plasma Physics and Controlled Fusion, vol. 47, no. 1, p. 155, 2005.

[15] M. D. Boyer, J. Barton, E. Schuster, T. C. Luce, J. R. Ferron, M. L. Walker, D. A. Humphreys, B. G. Penaflor, and R. D. Johnson, "Firstprinciples-driven model-based current profile control for the DIII-D tokamak via LQI optimal control," Plasma Physics and Controlled Fusion, vol. 55, no. 10, p. 105007, 2013.

[16] M. D. Boyer, J. Barton, E. Schuster, M. L. Walker, T. C. Luce, J. R. Ferron, B. G. Penaflor, R. D. Johnson, and D. A. Humphreys, "Backstepping control of the toroidal plasma current profile in the DIIID tokamak," IEEE Transactions on Control Systems Technology, vol. 22, no. 5, pp. 1725-1739, 2014.

[17] E. Maljaars, F. Felici, M. De Baar, J. van Dongen, G. Hogeweij, P. Geelen, and M. Steinbuch, "Control of the tokamak safety factor profile with time-varying constraints using mpc," Nuclear Fusion, vol. 55, no. 2, p. 023001, 2015.

[18] N. M. T. Vu, R. Nouailletas, L. Lefèvre, and F. Felici, "Plasma qprofile control in tokamaks using a damping assignment passivity-based approach," Control Engineering Practice, vol. 54, pp. 34-45, 2016.

[19] S. Kim and J. Lister, "A potentially robust plasma profile control approach for iter using real-time estimation of linearized profile response models," Nuclear Fusion, vol. 52, no. 7, p. 074002, 2012.

[20] F. Bribiesca Argomedo, C. Prieur, E. Witrant, and S. Brémond, "A strict control Lyapunov function for a diffusion equation with timevarying distributed coefficients," IEEE Transactions on Automatic Control, vol. 58, no. 2, pp. 290-303, 2013.

[21] A. Gahlawat, E. Witrant, M. M. Peet, and M. Alamir, "Bootstrap current optimization in tokamaks using sum-of-squares polynomials," in 51 st Annual Conference on Decision and Control, 2012, pp. 4359-4365.

[22] O. Gaye, L. Autrique, Y. Orlov, E. Moulay, S. Brémond, and R. Nouailletas, "H $\infty$ stabilization of the current profile in tokamak plasmas via an LMI approach," Automatica, vol. 49, no. 9, pp. 2795-2804, 2013.

[23] P. Kokotovic, H. K. Khalil, and J. O'reilly, Singular perturbation methods in control: analysis and design. SIAM, 1999, vol. 25.

[24] H. K. Khalil, Nonlinear systems, 3rd ed. Upper Saddle River, (N.J.): Prentice Hall, 2002.

[25] Z. Gajic, Optimal control of singularly perturbed linear systems and applications. CRC Press, 2001.

[26] R. Vazquez and M. Krstic, Control of turbulent and magnetohydrodynamic channel flows: boundary stabilization and state estimation. Springer Science \& Business Media, 2008.

[27] Y. Tang, C. Prieur, and A. Girard, "Tikhonov theorem for linear hyperbolic systems," Automatica, vol. 57, pp. 1-10, 2015.

[28] F. Felici and O. Sauter, "Non-linear model-based optimization of actuator trajectories for tokamak plasma profile control," Plasma Physics and Controlled Fusion, vol. 54, no. 2, p. 025002, 2012. 
[29] P. Geelen, F. Felici, A. Merle, and O. Sauter, "Parameter estimation for a nonlinear control-oriented tokamak profile evolution model," Plasma Physics and Controlled Fusion, vol. 57, no. 12, p. 125008, 2015.

[30] F. Hinton and R. Hazeltine, "Theory of plasma transport in toroidal confinement systems," Reviews of Modern Physics, vol. 48, no. 2, p. 239, 1976.

[31] F. Bribiesca Argomedo, E. Witrant, C. Prieur, S. Brémond, R. Nouailletas, and J. F. Artaud, "Lyapunov-based distributed control of the safety factor profile in a tokamak plasma," Nuclear Fusion, vol. 53, no. 3, p. 033005, 2013.

[32] W. Kaplan, Advanced calculus. Addison Wesley, 2002.

[33] J. Löfberg, "YALMIP: A toolbox for modeling and optimization in matlab," in IEEE International Symposium on Computer Aided Control Systems Design, Taipei, Taiwan, 2004, pp. 284-289.

[34] F. Bribiesca Argomedo, E. Witrant, and C. Prieur, "D 1-Input-to-state stability of a time-varying nonhomogeneous diffusive equation subject to boundary disturbances," in American Control Conference, Montreal, Canada, 2012, pp. 2978-2983.

[35] F. Felici, M. de Baar, and M. Steinbuch, "A dynamic state observer for real-time reconstruction of the tokamak plasma profile state and disturbances," in American Control Conference, Portland, USA, 2014, pp. 4816-4823.

[36] O. Sauter, C. Angioni, and Y. Lin-Liu, "Neoclassical conductivity and bootstrap current formulas for general axisymmetric equilibria and arbitrary collisionality regime," Physics of Plasmas, vol. 6, no. 7, pp. 2834-2839, 1999

[37] M. Erba, T. Aniel, V. Basiuk, A. Becoulet, and X. Litaudon, "Validation of a new mixed bohm/gyro-bohm model for electron and ion heat transport against the iter, tore supra and start database discharges," Nuclear Fusion, vol. 38, no. 7, p. 1013, 1998. 